\title{
High Temperature and High Hydrostatic Pressure Cultivation, Transfer, and Filtration Systems for Investigating Deep Marine Microorganisms
}

\author{
Gina C. Oliver ${ }^{1,2 ¥}$, Anaïs Cario ${ }^{1,3 * *}$, Karyn L. Rogers ${ }^{1,4 *}$
}

${ }^{1}$ Department of Earth and Environmental Sciences, Rensselaer Polytechnic Institute, Troy, NY, USA

${ }^{2}$ Current address: Department of Geology, San Bernardino Valley Community College, San Bernardino, CA, USA

${ }^{3}$ Current address: CNRS, Univ. Bordeaux, Bordeaux INP, ICMCB, F-33600, Pessac, France

${ }^{4}$ Rensselaer Astrobiology Research and Education Center, Rensselaer Polytechnic Institute, Troy, NY, USA

${ }^{¥}$ These authors contributed equally to this work

*Correspondence:

Corresponding Authors

rogerk5@rpi.edu

anais.cario@icmcb.cnrs.fr

Keywords: high-pressure microbiology, deep biosphere, cultivation devices, decompression

\begin{abstract}
High temperatures (HT) and high hydrostatic pressures (HHP) are characteristic of deepsea hydrothermal vents and other deep crustal settings. These environments host vast and diverse microbial populations, yet only a small fraction of those populations have been successfully cultured. This is due, in part, to the difficulty of sampling while maintaining these in situ conditions and also replicating those high-temperature and high-pressure conditions in the laboratory. In an effort to facilitate more HT-HHP cultivation, we present two HT-HHP batch culture incubation systems for cultivating deep-sea vent and subsurface (hyper)thermophilic microorganisms. One HT-HHP system can be used for batch cultivation up to $110 \mathrm{MPa}$ and $121^{\circ} \mathrm{C}$, and requires sample decompression during subsampling. The second HT-HHP system can be used to culture microorganisms up to $100 \mathrm{MPa}$ and $160^{\circ} \mathrm{C}$ with variable-volume, pressure-retaining vessels that negate whole-sample decompression during subsampling. Here, we describe how to build cost effective heating systems for these two types of high-pressure vessels, as well as the protocols for
\end{abstract}


HT-HHP microbial batch cultivation in both systems. Additionally, we demonstrate HHP transfer between the variable-volume vessels, which has utility in sampling and enrichment without decompression, laboratory isolation experiments, as well as HHP filtration.

\section{Introduction}

Deep-sea hydrothermal vents and subseafloor environments are highly productive ecosystems in the deep biosphere. With the average deep-sea vent depth at $\sim 2.1 \mathrm{~km}$ (i.e. $\sim 21 \mathrm{MPa}$; Von Damm, 1990), high-pressure conditions are inherent in these systems (Fry et al., 2008; Jebbar et al., 2015). The microbial communities in these environments are metabolically diverse and often display unique adaptive strategies that operate under elevated-pressure conditions. Currently only $\sim 0.1 \%$ of the microbial populations sampled from the subsurface are successfully cultured (D'Hondt et al., 2004, Parkes et al., 2014). In most cases, the isolates obtained from these environments are cultivated at in situ temperatures and $0.1 \mathrm{MPa}$, and their growth under elevated pressure conditions is rarely characterized. Therefore, our view of deep-sea vent and subseafloor microbial diversity is biased toward those microbes that are able to withstand drastic pressure changes compared to in situ conditions, and our understanding of microbial physiology and metabolism is largely based on growth at surface pressures (e.g. $0.1 \mathrm{MPa}$ ). This is primarily because high temperature (HT) and high hydrostatic pressure (HHP) microbial cultivation is not a widely adopted laboratory technique. HT-HHP microbial cultivation usually requires expensive equipment, specialized laboratory training, and it is more labor-intensive compared to ambient pressure experiments (Cario et al., 2019). However, HT-HHP cultivation is necessary if deep-sea and subsurface isolates are to be better-represented among the characterized isolated species. Furthermore, such laboratory techniques are critical to our understanding of pressure adaptation and potential unknown metabolic and physiological strategies relevant to biotechnology, geoengineering, medical sciences, and biogeochemical sciences. Therefore, we offer some practical HT-HHP batch culture experimental options to encourage more laboratories to adopt these longstanding techniques and expand the collective knowledge of how life thrives in deep-sea vents and in the subsurface.

High-pressure microbial batch cultivation is not a new idea and variations of vessels and culturing containers have been implemented over the last 70 years (Schwarz et al.; 1976; Jannash et al., 1980; Deming and Colwell, 1981; Yayanos, 1986; Zobell and Oppenheimer, 1950; Jannasch et al., 1966; McNichol et al., 2016 \& 2018). The same initial HHP batch cultivation techniques described in Zobell and Oppenheimer (1950) are still in use today (e.g. Orcutt et al., 2008; this study), and are also easily modified to accommodate other vessel configurations or experimental requirements. For example, vessels used for batch cultivation with sample decompression described in Yayanos $(1982 ;$ 2001) can be fabricated at a lower cost when purchased in bulk. Microorganisms in fluid medium or sediment can be grown in these types of vessels at HT-HHP using a variety of cultivation techniques in syringes (Baross \& Deming, 1983; Marteinsson et al., 1999; Takai et al., 2008), glass tubes with rubber stoppers (Bowles et al., 2011; Tasumi et al., 2015), heat sealed plastic bags (Berger et al., 1970), and sealed plastic pipette bulbs (Delong and Yayanos, 1986; Marietou et al., 2015). For HT-HHP systems that negate whole sample decompression, custom modifications can be made to vessels, like the PUSH vessels described below, to also cultivate sediment samples. Currently the PUSH vessels are designed for microbial batch cultivation in a fluid medium

Here we present two effective systems for routine HT-HHP batch cultivations. The first HT-HHP batch-culturing system was designed and built to accommodate four static pressure 
vessels, where sample decompression and repressurization is required for subsampling. Even though decompression-repressurization cycles can be detrimental to microbial growth (Oliver et al., 2020), the effects of decompression can be experimentally measured and the experimental design can be modified to minimize the decompression-repressurization cycles (e.g. Yayanos 1995; Yayanos, 2001). For example, additional HHP vessels can be used so that samples are only exposed to a single decompression-repressurization cycle, or samples can be fixed prior to decompression (i.e. Marietou et al., 2015). The second system we describe was built for variablevolume, floating-piston vessels that allow for subsampling without decompression. These pressurized underwater sample handler (PUSH) vessels, based on previous designs (Bianchi et al., 1999, Tamburini et al., 2003), were purchased through TOP Industrie ${ }^{\odot}$ and designed with a polyether ether ketone (PEEK) internal reservoir with a floating piston that maintains system pressure during subsampling via a connection to a HHP pump. Furthermore, the PUSH vessel HTHHP system was built for mobility to allow for anaerobic preparations in an anaerobic chamber without heat loss so that stable pressures could be achieved quickly. In general, batch cultivation is a common and effective microbiological technique used to explore microbial growth, metabolism, and physiology (e.g. Kell, 1987; Maier, 2000). In the HT-HHP systems presented here, such investigations can be extended to a range of temperature, pressure, and geochemical conditions that better replicate a variety of deep-sea vent and subsurface environments. These systems have been applied to the cultivation of two extremophiles, Archaeoglobus fulgidus and Desulfovibrio salexigens (Oliver et al., 2020), and similar systems have been used in other deepocean settings (Garel et al., 2019).

\section{HT-HHP system in static pressure vessels}

Different pressure retaining vessels for microbial sample collection from the deep sea and HT-HHP cultivation have been designed over the last fifty years (reviewed in Tamburini, 2006), and a few of those designs were specifically made to cultivate hyperthermophilic piezophiles (Kyo et al., 1991; Cangenella et al., 1997; Kato, 2006; Parkes et al., 2009). Here we present a configuration for using several common static high-pressure vessels in parallel with a heating system that allows for batch cultivation of (hyper)thermophilic microorganisms at elevated pressures.

\subsection{Design objectives and schematics}

The objective for this HT-HHP cultivation system was to build an installation where several (4) pressure vessels could be secured in place, heated, insulated, and pressurized by a single HHP pump. Each vessel required a temperature-controlled system, a pressure line to the pump, and an easily attachable and mobile pressure gauge (Fig. 1). Four vessels were included in the setup to facilitate triplicate experiments with a single negative control. Finally, the length, width, and height of this system design considered table space availability and usability by researchers. 


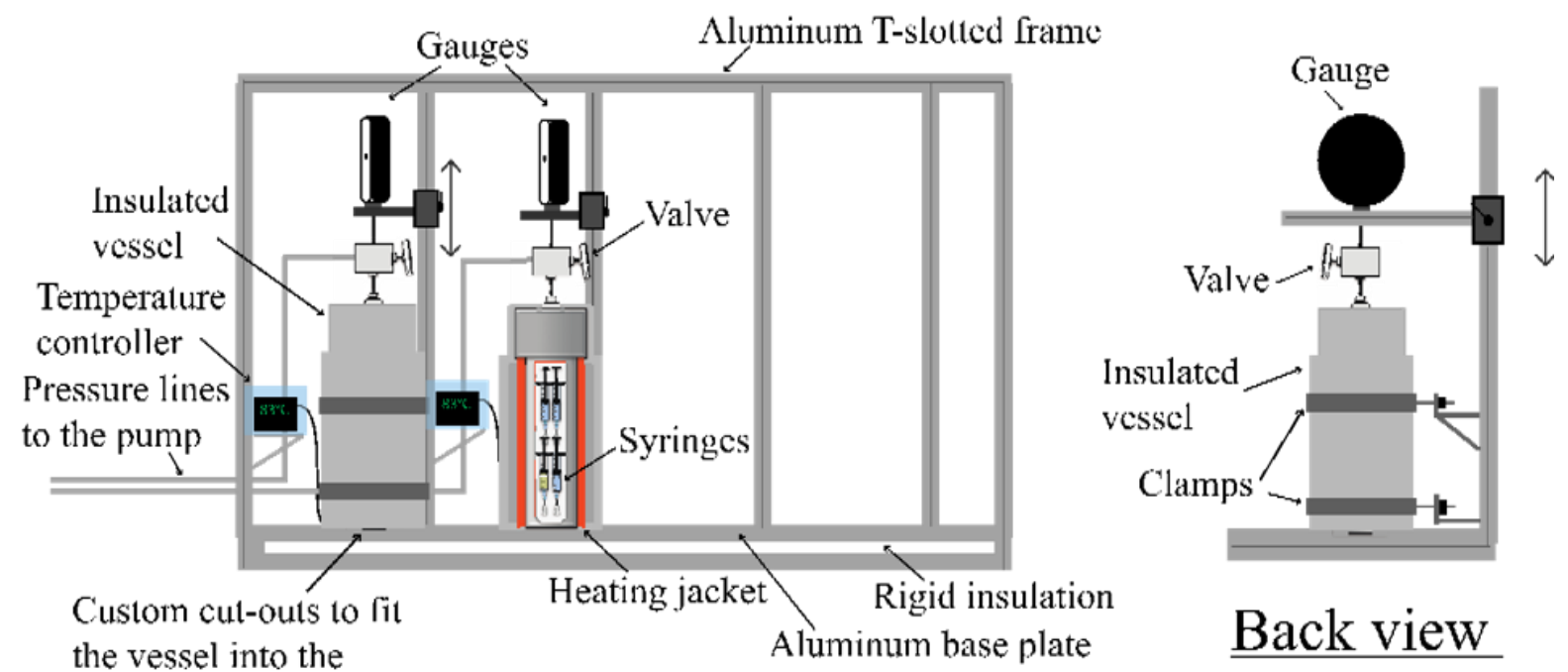

base plate

\section{$\underline{\text { Side view }}$}

Figure 1. A design schematic of the HT-HHP system installation for static pressure vessels. The side view shows an insulated vessel and a cross section of an insulated vessel to show the heating element and syringe sample loading. The back view highlights the adjustable gauge and vessel security.

\subsection{Vessels and Thermal Control}

Four $125 \mathrm{~mL}$ static volume pressure vessels from High Pressure Equipment Co. ${ }^{\odot}\left(\mathrm{HiP}^{\odot}\right.$, OC-1 O-Ring series) were adapted for HT/HHP batch cultivation. Temperature was controlled via a silicone rubber heating jacket together with CNi16D33 temperature controllers (OMEGA) and a type $\mathrm{J}$ thermocouple. Thermocouples, heating jackets, and power cord wires were attached to the temperature controllers by terminal connections according to the OMEGA ${ }^{\mathrm{TM}}$ instructions manual for the CNi16D33 temperature controllers. Thermocouples were first calibrated in a $0^{\circ} \mathrm{C}$ ice bath and the controllers were then set to the desired temperature for each experiment (e.g. $83^{\circ} \mathrm{C}$ for the experiments described below) and auto-tuned. After auto-tuning, fine calibration was done following the OMEGA ${ }^{\mathrm{TM}}$ manual and the set temperature was maintained within $\pm 0.5^{\circ} \mathrm{C}$. Temperature and pressure limits were based on the maximum working capacities of the $\mathrm{HiP}^{\odot}$ vessels and OMEGA ${ }^{\mathrm{TM}}$ heating jackets (see below).

\subsection{Materials}

The installation frame was constructed from $80 / 20^{\circledR}$ Inc. T-slotted aluminum framing and accessories. T-slotted framing profiles, the 31-inch long by 9 -inch wide aluminum base plate, nuts, bolts, fasteners, mounts, and brackets were purchased from $80 / 20{ }^{\circledR}$ Inc. Clamping hangers, rigid galvanized standard duct, insulation (fiberglass and high temperature calcium silicate blocks), screws, fuses, power cords, and clear Nema boxes were purchased from McMaster Carr. Temperature controllers (CNi16D33), silicone rubber heating jackets, thermocouples (type J) were purchased from OMEGA ${ }^{\text {TM }}$. High pressure gauges, gauge hex nut reducer, $1 / 4$ inch medium pressure tubing, valves and connections were purchased from Swagelok ${ }^{\circledR}$, all rated to $137.89 \mathrm{MPa}$.

\subsection{Construction}


The vessel installation frame was constructed first (Fig. 1). Because the bottom of each vessel was slotted to fit into a vice (for torque during lid closure or removal) a custom-mahcined aluminum baseplate was needed to fit the base shape of each vessel. This allowed for vessel stability when additional torque was needed to open the vessels. Rigid calcium silicate insulation was placed under each baseplate cutout to insulate the bottom of each vessel and additional insulation was placed underneath the entire baseplate. Despite these measures, small areas of the stainless steel vessels were in contact with the aluminum base plate resulting in minor heat loss. This issue is addressed in section 2.5 .

The installation frame was assembled and bolted to the lab bench (Fig. 2). Each pressure vessel was placed into a cutout on the baseplate. Temperature was monitored by thermocouples ( $\mathrm{J}$ type) attached on the outside of each vessel and the thermocouples were connected to a temperature controller $\left(\mathrm{OMEGA}^{\mathrm{TM}}\right)$ that was calibrated and programmed. Each vessel was then wrapped in a 7-inch $\mathrm{x} 10$-inch silicone rubber heating jacket $\left(\mathrm{OMEGA}^{\mathrm{TM}}\right)$ and insulated (Fig. 1). Heating jackets and insulation were wrapped around each vessel with hook and loop fasteners. To further stabilize each static pressure vessel, five-foot rigid standard duct was cut into one-foot lengths and placed over the vessel heating jacket and insulation. The vessels were then secured to the aluminum frame by wrapping two threaded rod-clamping hangers around the rigid duct (Fig. 1).

Each vessel was equipped with a pressure gauge that was secured on an arm attached to the main installation frame. The extension arms can be adjusted vertically so that the gauges can be connected and disconnected from each vessel during lid removal. The high-pressure gauge was fitted to the extended arm by a hex nut connection. The hex nuts were machined to fit into the $80 / 20^{\circledR}$ Inc. mounts and were then fastened to the t-slotted extension arm off the main t-slotted frame. The four extension arms for each pressure gauge were connected to the main frame with single mount Unibearing ${ }^{\mathrm{TM}}$ assemblies. The pressure gauges were connected to a three-way valve: one way to the vessel, one way to the pressure gauge, and one way to the high-pressure line. Four high pressure lines were built for each vessel from $1 / 4$ inch high-pressure stainless steel tubing $\left(\right.$ Swagelok $\left.{ }^{\circledR}\right)$ that was measured, cut, bent, coned, and threaded so that each vessel could be connected to the high pressure screw pump rated to $206 \mathrm{MPa}\left(\mathrm{HiP}^{\odot}\right.$, Fig. 2).

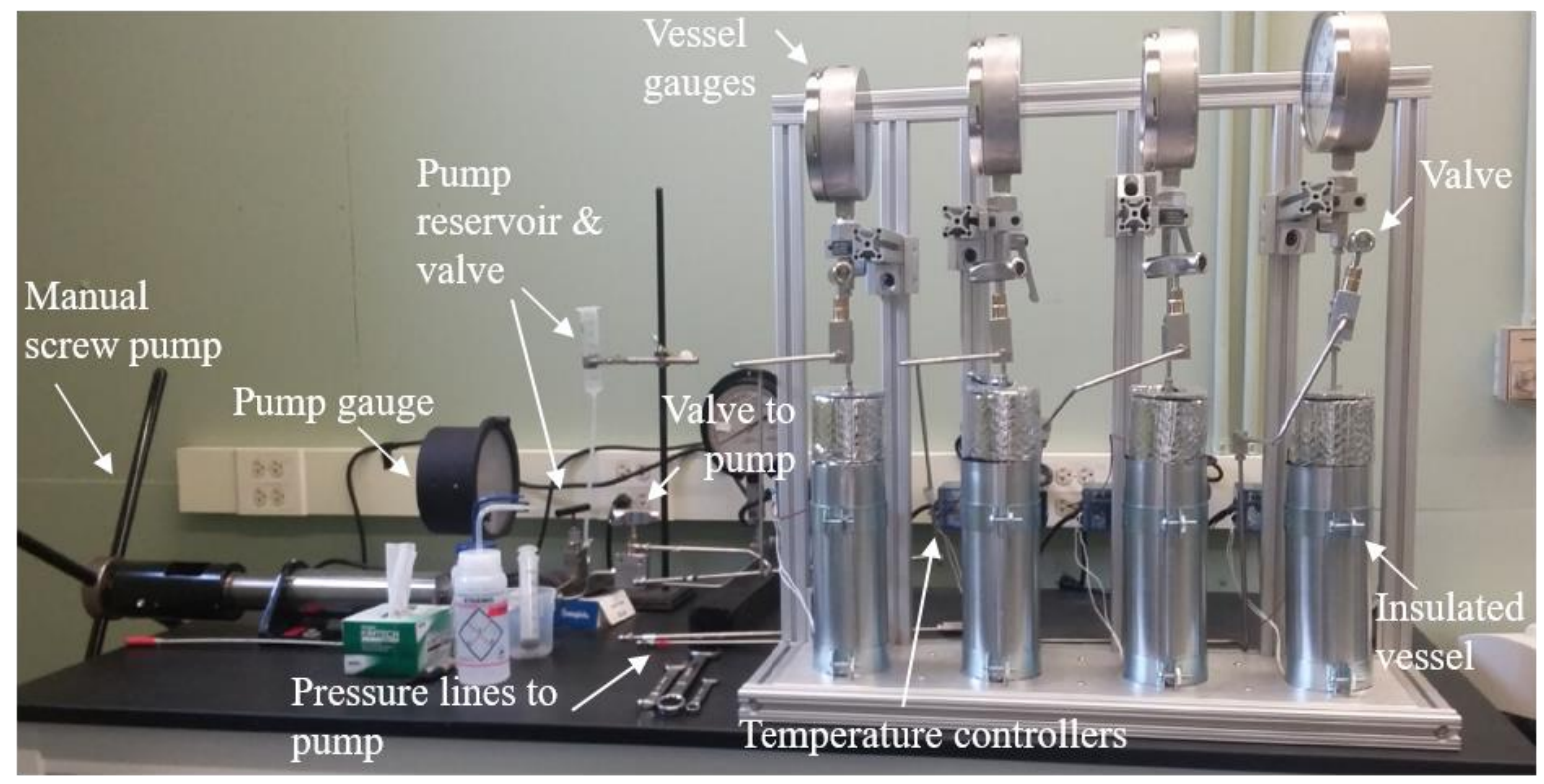


Figure 2: Picture of the HT-HHP system for static pressure vessels with temperature-controlled, insulated vessels.

\subsection{Equipment testing}

Following initial assembly, and prior to every experiment, the complete experimental setup was pressure-tested, O-rings were inspected and lubricated as necessary, and the temperature control system calibration was verified. The high-pressure lines were regularly checked for leaks before and during HHP experiments. Internal vessel temperatures were monitored by thermometer and the controllers were set to the desired internal vessel temperature. Since there were small areas of the vessel in direct contact with the aluminum base, heat was lost at the bottom of the vessels, resulting in a small temperature gradient that left the bottom two inches of the vessel $\sim 4-6^{\circ} \mathrm{C}$ cooler than the set temperature that was obtained in the main body of the vessel. Therefore, samples were always placed above this thermal decline. Mitigation of this thermal gradient requires extension of the heating jacket and/or additional insulation around the base of the vessel.

\subsection{Batch cultivation in syringes}

Various methods of HHP cultivation in static pressure vessels have been reviewed by Yayanos (2001), and many focus on the growth of heterotrophs in stoppered-syringes, usually custom made. Methods described in Takai et al., (2008) and Tasumi et al., (2015) were developed for autotrophic HHP batch cultivation of anaerobes in gas-tight, glass syringes, which could also be applied to anaerobic heterotrophs. In general, plastic syringes can be used for HHP heterotrophic growth of facultative anaerobes or anaerobic sulfate reducing microorganisms (Yayanos, 2001). The advantages of plastic syringes for HHP growth is that they are cost effective and several will fit in most pressure vessels to facilitate experimental replication. However, because plastic syringes are not gas tight, small amounts of oxygen can penetrate the culture medium. In strains grown in a medium containing $\mathrm{Na}_{2} \mathrm{~S}$ to maintain anoxif and/or strains that produce sulfide, oxygen stress can be mitigated. For species particularly sensitive to oxygen, glass, gas-tight syringes will prevent oxygen contamination. Cell densities and cell morphology can be indicative of oxidative stress, and should be compared between syringe types to check for oxidative stress.

\subsection{Experimental Design and Protocol}

Design of a HT-HHP growth experiment in static pressure vessels using syringes as microbial growth reactors has to balance the growth rate, the sampling interval, and the number of syringes that can fit into each static pressure vessel, while minimizing the decompression and repressurization cycles. In general, to conduct an experiment in triplicate with a single negative control, each of the four vessels will contain one syringe from each triplicate and a negative control syringe. Subsampling required to construct a robust growth curve will cycle through all four vessels so that each vessel and the syringes contained therein are only decompressed and repressurized at every fourth sampling interval. The protocol below follows this design, but can be easily modified depending on alternative experimental requirements, which might require additional vessels, syringes, or alternative reaction volumes

\subsubsection{Preparation of growth media and pre-cultures}

In general, cultivation in syringes begins with preparation and inoculation of the growth medium in serum bottles followed by transfer into syringes. The methods described here are 
designed specifically for the growth of anaerobes and include several steps to prevent oxygen contamination while maintaining sterility. These protocols could be simplified if anoxia is not required. A sterile growth medium appropriate for the microbial species of interest should be prepared in sterile serum bottles, and the number of serum bottles should be equivalent to the number of syringes needed for the entire experiment. In the case described here, thirteen serum bottles containing sterile anoxic growth media were required for triplicate growth experiments in four vessels with one negative control (Fig. 3A), in addition to the vials required to obtain an actively growing culture from frozen stock, as well as the growth of pre-cultures. The volume of media in each bottle should be twice the target syringe volume, so that the remaining inoculated volume can be incubated at ambient pressures as a positive growth control. Finally, pre-cultures are prepared from frozen stocks so that logarithmic phase is achieved just prior to inoculation of the experiment.

1. Prepare sterile media appropriate for the target species. If anoxia is required, boil the medium under $\mathrm{N}_{2}$ and adjust $\mathrm{pH}$ as required.

2. Distribute $10 \mathrm{~mL}$ anoxically into serum bottles under $\mathrm{N}_{2}$; cap and crimp, then autoclave for 15 minutes at $121^{\circ} \mathrm{C}$ for sterilization (Balch et al., 1979).

3. Following sterilization, anoxic conditions can be maintained by adding $0.1 \mathrm{~mL}$ sulfide from a $2.5 \%\left(\right.$ w/v) anaerobic solution $\left(\mathrm{Na}_{2} \mathrm{~S} * 9 \mathrm{H}_{2} \mathrm{O}\right)$ for every $10 \mathrm{~mL}$ of medium, or otherwise as required for the target species.

4. Inoculate sterile growth medium with frozen stock of the target species. In general $1 \%$ inoculation volume will allow for a robust logarithmic phase of growth.

5. Use logarithmic phase aliquots of this culture as inoculum for three pre-cultures, one for each of the triplicate HT-HHP batch growth experiments. Incubate at the desired temperature. Monitor growth and measure cell density prior to inoculation of experimental serum bottles.

\subsubsection{Syringe preparation, inoculation and transfer}

Pre-cultures prepared as described in section 2.7.1 are used to inoculate the sterile growth medium in serum bottles, which is immediately transferred to sterile and anoxic syringes. These are then moved into pre-heated pressure vessels and pressurized. Again, additional steps are included here to maintain anoxia in the growth medium and these steps could be excluded for the growth of aerobes. Details below are for a triplicate growth experiment conducted in $5 \mathrm{~mL}$ syringes alongside a negative control conducted in $3 \mathrm{~mL}$ syringes. Each triplicate of the positive growth experiment and the negative control are distributed in 4 syringes that are incubated in separate pressure vessels to minimize decompression/repressurization cycles. To complete a triplicate batch cultivation experiment 16 syringes were prepared. Twelve $5 \mathrm{~mL}$ syringes were used as positive growth experiments and four $3 \mathrm{~mL}$ syringes were used for negative controls (Figs. 3A and 3B).

1. Preheat the pressure vessels filled with ultrapure water $(18.2 \mathrm{M} \Omega)$ to the desired experimental temperature. Be sure to open at least one valve so that the temperature increase does not increase the pressure during pre-heating.

2. While maintaining sterility, fit twelve 5-mL and four 3-mL Becton-Dickinson (BD) plastic syringes with 23-gauge BD needles.

3. Flush each syringe with $\mathrm{N}_{2}$ 3-4 times. This can be done by inserting each syringe into a sterile, $\mathrm{N}_{2}$-filled stoppered flask, retracting the plunger to fill the syringe with $\mathrm{N}_{2}$, removing the syringe from the flask and pushing out the gas while maintaining sterility.

4. With the syringe full of $\mathrm{N}_{2}$, immediately embed the needle partway in the middle of a sterile butyl rubber stopper or silicone stopper. 
5. Inoculate 3 sets of 4 serum bottles each with one of the three pre-cultures to the desired cell density. Depending on growth rate and measurement technique either a $1 \%(\mathrm{v} / \mathrm{v})$ inoculum volume or a target cell density of $\sim 10^{6} \mathrm{cells} / \mathrm{ml}$ is ideal.

6. Shake each serum bottle to evenly distribute the cells.

7. Immediately transfer $5 \mathrm{~mL}$ of inoculated growth medium from each serum bottle into each syringe using sterile techniques. Ideally, transfers are conducted in an anaerobic chamber. To maintain anoxia, remove the stopper from the needle, expel the last of $\mathrm{N}_{2}$ from the syringe while inserting the needle into the serum bottle stopper. Fill the syringe to slightly over $5 \mathrm{~mL}$, and reduce to $5 \mathrm{~mL}$ before re-inserting the needle into the silicone stopper. This should produce 12 total inoculated 5-mL syringes, 4 of each of the three pre-cultures.

8. For the negative controls, using the same anoxic and sterile techniques transfer the sterile growth medium from the serum bottle into four, 3-mL syringes.

9. Distribute the syringes into the four pre-heated vessels as described above (Fig. 3).

10. Pressurize each vessel to the desired experimental pressure.

11. Incubate the remaining inoculated fluid in each of the serum bottles at the experimental temperature as a control of cell viability. Note that this is only appropriate for cells able to grow at ambient pressures.

From preculture \#
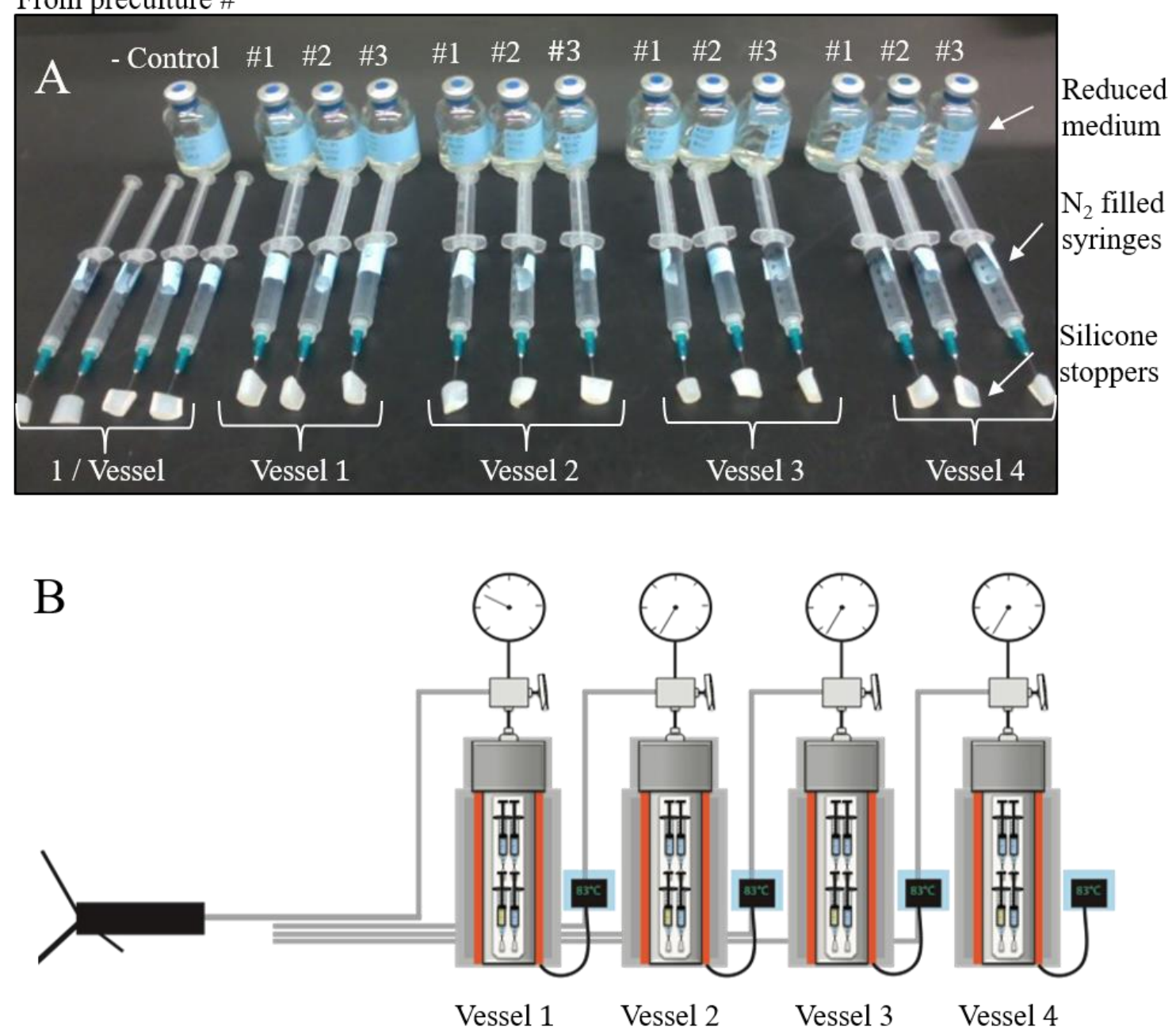
Figure 3: (A) A picture of serum bottles filled with reduced sterile medium for inoculation from three separate pre-cultures and flushed plastic syringes prepared for anaerobic transfer of inoculated medium. (B) A schematic of the four heated pressure vessels with syringe samples. This illustrates an example of a HTHHP growth curve experiment done in triplicate with syringe distribution into each vessel.

\subsubsection{Sampling with decompression}

In order to quantify cell density as a function of time each growth experiment was subsampled at regular intervals. A subsampling interval that produces 8-12 data points distributed across the lag-, logarithmic, and stationary-growth phases is optimal. However, the time between decompression and repressurization must also be maximized. Each vessel contained one representative from each triplicate and the negative control, and only one vessel was used per sampling interval. Therefore, each set of experimental syringes (one from each pre-culture and the negative control) was only decompressed and repressurized every fourth sampling interval. Here we describe subsample preparation for subsequent quantification of cell density via epifluorescence microscopy.

1. At each sampling interval, decompress one vessel at an average rate of $19 \mathrm{MPa} / \mathrm{minute}$ or slower. Some microorganisms are more sensitive to decompression that others and a slower decompression rate may be required.

2. Remove the syringes and fix a $0.5 \mathrm{~mL}$ subsamples in $2.5 \%$ gludaraldehyde and store at $4{ }^{\circ} \mathrm{C}$ until analysis.

3. Return the syringes to the heated vessels and repressurize.

\section{Pressurized Underwater Sample Handler (PUSH) vessel HT-HHP system}

The use of the PUSH system for laboratory cultivation of extremophiles leverages the variable-volume, floating-piston design of these vessels to achieve HT-HHP batch culture experiments that have no decompression/repressurization cycles during subsampling. While the initial motivation for developing these vessels was to retrieve water column samples at in situ pressures (Bianchi et al., 1999, Tamburini et al., 2003), coupling these vessels to other isobaric samplers and to each other enables fully isobaric sample processing, including sample transfer, enrichment, and filtration. Here we describe a mobile HT-HHP system built for eight pressureretaining vessels with reservoir sizes of $50 \mathrm{~mL}$ for batch culture to facilitate post-sampling HTHHP enrichment and transfer experiments.

\subsection{Design objectives and schematics}

The objectives for the second HT-HHP cultivation system design were to build portable heating systems that could be taken on and off of the PUSH vessels, create a portable power source for the heating systems to heat the vessels in an anaerobic chamber, and build an installation frame for eight vessels so that each vessel could be pressurized from one or two hydraulic pump(s). Overall, this heating system needed to be portable, removable, and suitable for continually heating the vessels in the anaerobic chamber. Again, the length, width, and height of this system design considered table space availability and usability by researchers.

\subsection{Vessels}

The pressurized underwater sample handler (PUSH) vessels were made by TOP Industrie ${ }^{\odot}$ and have a maximum pressure and temperature range of $0.1-100 \mathrm{MPa}$ and $25-160^{\circ} \mathrm{C}$, respectively. Each vessel includes a stainless steel vessel body, a polyether ether ketone (PEEK) reservoir with 
a floating piston and lid, two screw caps (one screw cap for the PEEK piston side and one screw cap to the PEEK reservoir side), two valve connections and two valves (Figs. 4A-C). To preserve the lifetime of the PEEK reservoir, the working pressure range is suggested for use up to $80 \mathrm{MPa}$ (TOP Industrie $^{\odot}$ ). Performance is optimized, and screw cap seizing is prevented, when an antiseizing agent is used on the screw caps during routine batch laboratory culturing.
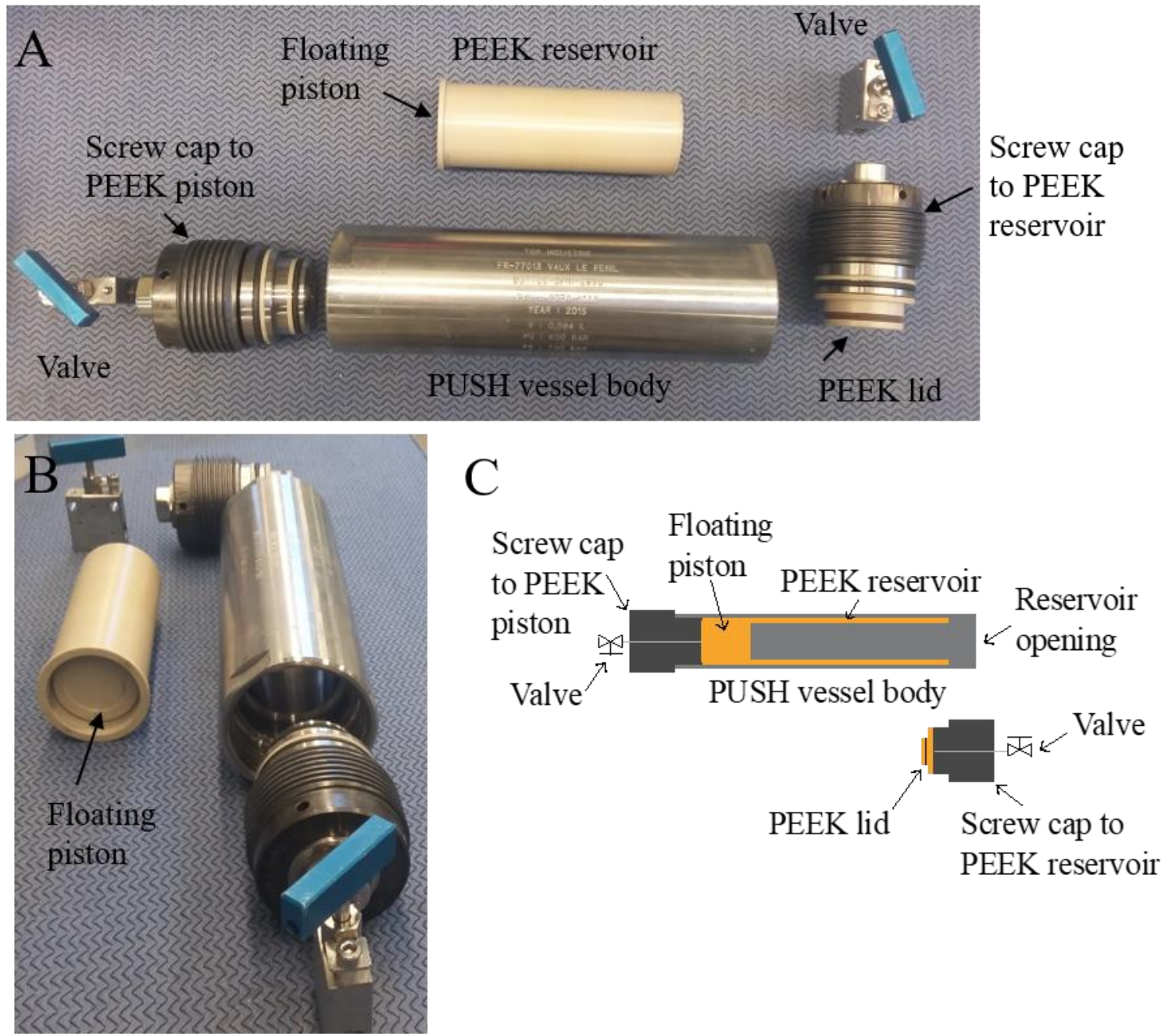

Figure 4: Picture of a disassembled Pressurized Underwater Sampler Handler (PUSH) vessel and individual parts (A), a side view of the PUSH showing the floating piston in the PEEK reservoir (B), and a schematic of the PUSH vessel (C).

\subsection{Materials}

The PUSH installation frame was also constructed from $80 / 20^{\circledR}$ Inc. T-slotted aluminum framing (Fig. 5). Clamping hangers, L-brackets, hinges, latches, clear Nema boxes (Fig. 5), rigid galvanized standard duct, insulation (flexible foam sheets and bubble insulation), 12-volt rechargeable large cell battery, battery charger, electrical wires, screws, fuses, and power cords were purchased from McMaster Carr. Eight additional temperature-controlled OMEGA ${ }^{\circledR}$ systems previously described in section 2.2 and 2.3 were purchased and assembled for up to eight PUSH vessels. The only differences in the heating system were the heating jacket size $(7 \times 7$ in. silicone rubber heaters) and the insulation (see section 2.4). Flexible 1/16-inch tubing and 1/16 to 1/8 inch 
adapters were purchased from $\mathrm{HiP}^{\odot}$ to make a high-pressure line that could connect the pump to each vessel. Also, 1/8 inch high-pressure tubing, one needle valve (HiP), and one micrometering valve (TOP Industrie ${ }^{\odot}$ ) were purchased to construct a decompression line.

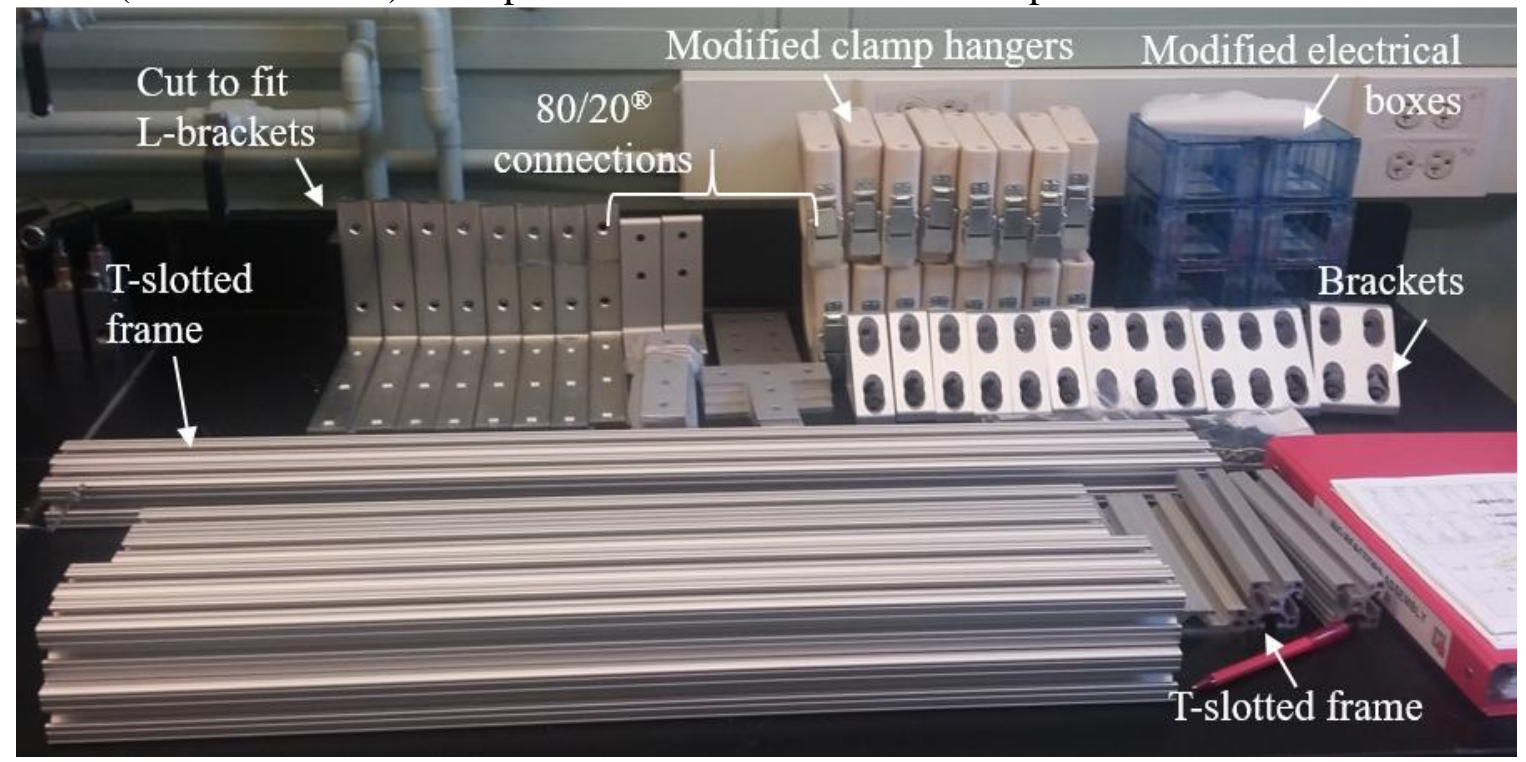

Figure 5: Picture of the HT-HHP installation system parts showing the T-slotted frame, connections, and brackets from $80 / 20 \AA$ and modified materials from McMaster Carr.

\subsection{Construction}

Modifications were made to most of the materials to fit the installation design (Fig. 5). To make supports and enclosures for each vessel, the following modifications were made: $8 \times 8$ inch L-brackets used to support the vessels were cut down to 4.5 inches on one side to fit the frame; hinges and latches were screwed into the clamping hangers; and eight 7-inch cylinders cut from a 10-foot stainless steel standard duct were cut in half lengthwise and deburred. Each half of the stainless steel duct cylinder was screwed onto each side of the clamping hanger, and the clamping hanger was bolted to the L-bracket. Each vessel enclosure was made from two clamping hangers bolted to a set of L-brackets (Fig. 6). The Nema electrical boxes were cut to fit the temperature controllers and holes were drilled through the boxes to enclose the heating and power element wires. Mounts for the Nema electrical boxes were constructed by attaching $4 \times 4$ inch L-brackets to the main T-slotted frame, then attaching 2x2 inch L-brackets to the $4 \times 4$ inch L-brackets. Finally, the installation was made up of two vertically stacked sections of four vessels (Fig. 6).

For the vessel heating system, the thermocouple was placed onto the middle of a PUSH vessel, then the heating jacket and insulation was wrapped around the vessel and thermocouple and was secured with two hook and loop fasteners. Each vessel had a double insulation wrap made from a flexible foam insulation sheet sealed to a less flexible bubble insulation sheet (Fig. 6). These heating wraps were made to be taken on and off of vessels. To power the heaters in the anaerobic chamber, a DC to AC power converter was purchased and connected to a 12-volt rechargeable lead-acid battery with a fuse attached to the positive cable (Fig. 7).

Finally, the high-pressure line and decompression line were assembled. The decompression line was made to minimize shear stress caused by a pressure differential during subsampling. This was made from using 1/8-inch high-pressure tubing and connecting it to a needle valve $\left(\mathrm{HiP}^{\odot}\right)$ and to a micrometering valve (TOP Industrie ${ }^{\odot}$ ). The high-pressure line was made with flexible 1/16- 
inch high-pressure stainless steel tubing and was connected to a 1/16-inch to $1 / 8$-inch adapter on both ends of the tubing, to accommodate the 1/8-inch connections on the PUSH vessels and pump. A plastic Luer-lok valve was placed at the end of the decompression line to connect any slip tip or Luer-lok syringes for sampling cell cultures.

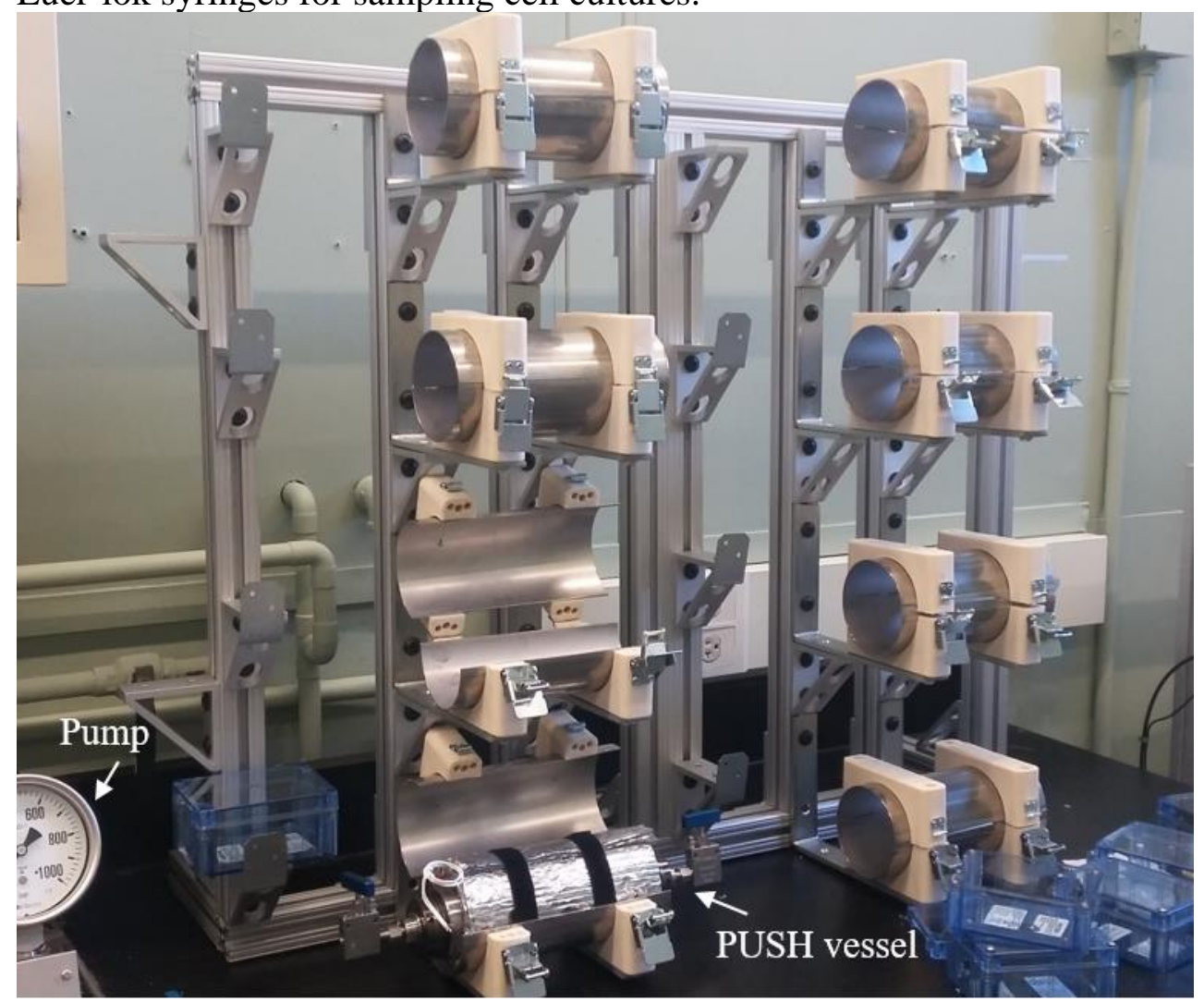

Figure 6: The main assembly of the HT-HHP installation frame with cut-to-fit stainless steel vessel holders, and for reference a hydraulic screw pump is shown in the bottom left and a PUSH vessel wrapped in a heating jacket and insulation is shown bottom center. 


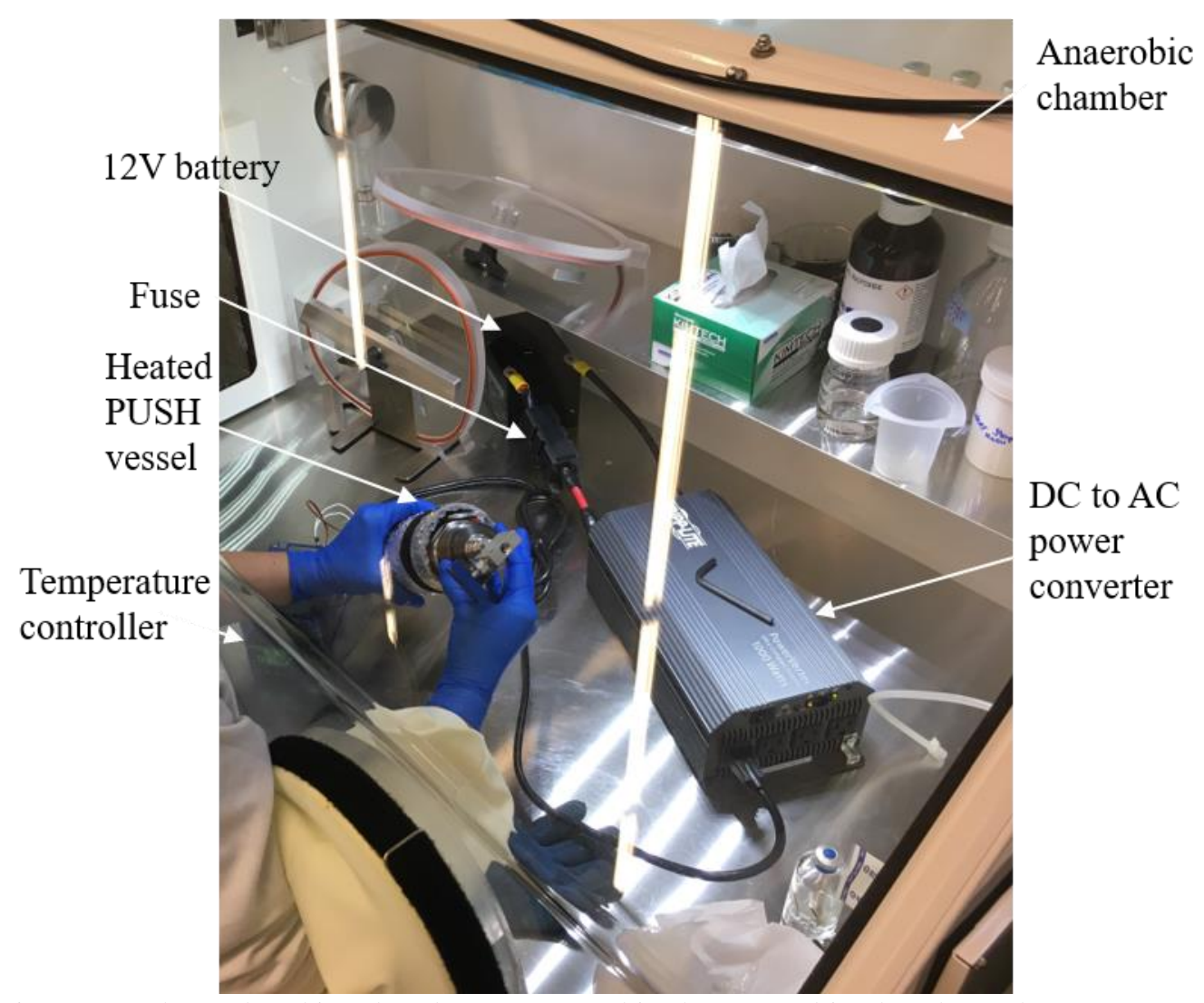

Figure 7: A heated and insulated PUSH vessel in the anaerobic chamber. The PUSH vessel and temperature controller system is plugged into the DC to AC power converter ran by a 12 -volt battery.

\subsection{Equipment testing}

As with all HHP experiments, the entire system was pressure-tested prior to each experiment, and connections and lines were leak-checked every time a new connection was made. At the start of each experiments, all O-rings were inspected and lubricant was added as needed, and anti-seizing agents were applied to stainless-steel threading. Finally, the temperature control system was calibrated. The internal PUSH vessel temperatures were monitored by directly measuring the ultrapure water $(18.2 \mathrm{M} \Omega$ ) fluid temperature inside the heated vessels with a Type $\mathrm{J}$ thermocouple. Only minimal temperature fluctuations were observed during cycling of the temperature controller. The controllers were then set to the desired internal vessel temperature for the experiment. When experiments require elevated temperatures (e.g. thermophile growth) the PUSH vessel and sterile medium need to be pre-heated prior to inoculation and pressurization. If enrichments or batch culture experiments for (hyper)thermophiles are started at room temperature and then pressurized and heated to a set temperature, the subsequent pressure increase that accompanies the temperature increase will need to be mitigated via a back-pressure valve or via continuous adjustment with the HHP pump. For the case of pre-heating the growth medium and vessels, we monitored temperature in the medium during the transfer from the anaerobic chamber and re-heating on the frame. A maximum temperature loss of $15^{\circ} \mathrm{C}$ was observed and the set temperature $\left(83^{\circ} \mathrm{C}\right)$ was reestablished within five minutes.

\subsection{Batch cultivation in PUSH vessels}


Design of a HT-HHP growth experiment in PUSH vessels has to balance the growth rate, the sampling intervals, sample loss in the decompression line, number of available vessels, and limited sample volume in the vessel reservoir. Microbial cultivation in PUSH vessels is significantly different than cultivation in static pressure vessels because each PUSH vessel is a single microbial growth reactor and requires additional preparation and maintenance. In PUSH vessels, culture medium comes in contact with parts of the stainless-steel vessel and special attention is needed to maintain sterility, and in desired cases, anoxia. Again, the methods described here are designed specifically for the growth of anaerobes and include several steps to prevent oxygen contamination while maintaining sterility. If anoxia is desired, inoculation and medium transfer into the PUSH vessels must be performed in an anaerobic chamber.

\subsubsection{PUSH vessel preparation}

In general, cultivation in PUSH vessels begins with sterilizing and pre-heating vessels. The vessels are partially assembled before placing into the autoclave and further assembled after sterilization. Vessels must be pre-heated before being pressurized to prevent drastic pressure fluctuations during the heating process. Four PUSH vessels were used to run a triplicate growth experiment with a negative control. Three vessels contained inoculated growth media and one vessel was filled with sterile growth media as a negative control.

1. Partially assemble each vessel before placing into the autoclave for sterilization. Insert the PEEK reservoir with the floating piston into each vessel and connect the screw cap to piston side and tighten to the vessel.

2. Place foil over the opening to the reservoir and wrap foil around the screw cap attached to the PEEK reservoir and attached PEEK lid, the valve opening on the PUSH vessel, and all valve connections and seal the foiled parts with autoclave tape.

3. Autoclave the PUSH vessels and all parts for 15 minutes at $121^{\circ} \mathrm{C}$.

4. After sterilization, assemble each PUSH vessel in a biosafety cabinet (Fig. 8A). This is done by partially screwing on the screw cap to PEEK reservoir to the main PUSH vessel. Partial closure aids in maintaining sterility when handling outside of the biosafety cabinet. Do not tighten the screw cap to the PEEK reservoir at this step, otherwise the PEEK lid will be sealed to the open reservoir (Fig. 8B), which needs to be left open for filling the vessel with the growth medium. Attach valves to both sides of the PUSH vessel and close the valve on the PEEK reservoir side was closed until transport to the anaerobic chamber. Leave the valve on the piston side open while pre-heating the vessels (Fig. 8B).

5. Take each vessel out of the biosafety cabinet and wrap each vessel and place a J-type thermocouple on the middle exterior section of the vessel. Then wrap each vessel and thermocouple in a heating jacket and insulating wrap (Fig. 8C).

6. Place vessels on the main installation frame and plug in the temperature controllers to start preheating. Once the vessels reach the desired incubation temperature, unplug and transported the vessels and attached heating systems into the anaerobic chamber. 


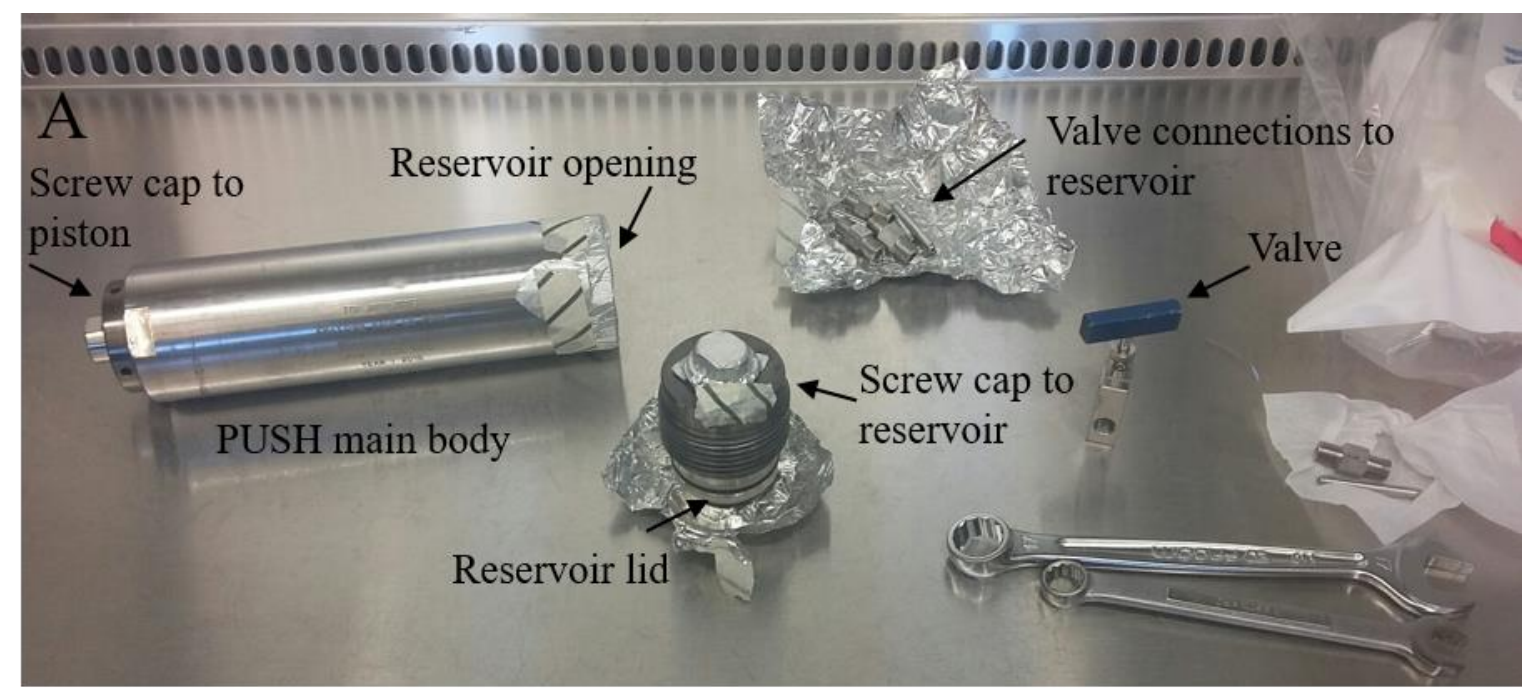

B
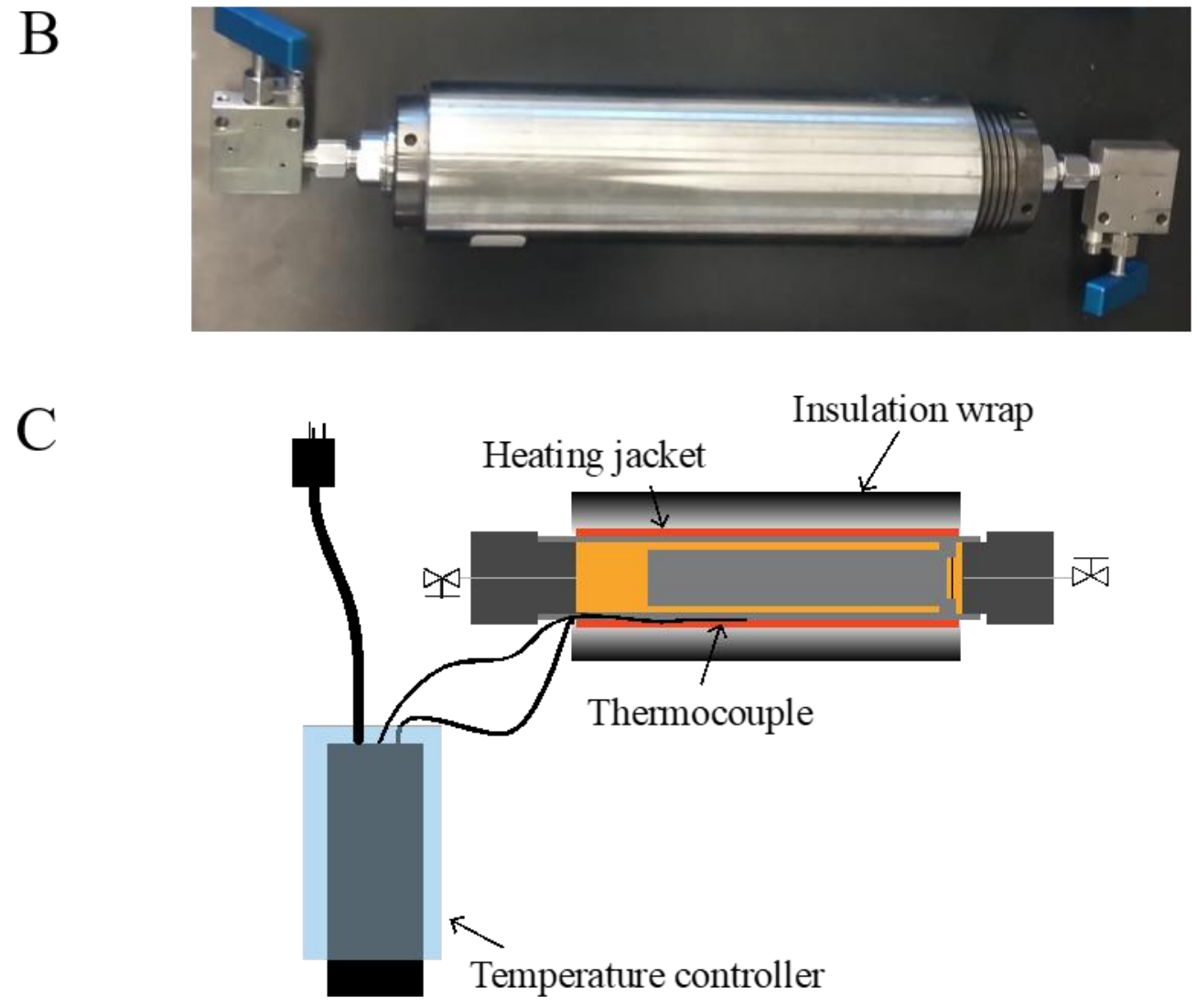

Figure 8: Picture of a sterile PUSH vessel and individual parts in a biosafety cabinet (A) for vessel assembly under sterile conditions. (B) An assembled PUSH vessel with the screw cap to the PEEK reservoir left partially unthreaded to later open for sample loading. (C) A schematic of a heated and insulated PUSH vessel with the temperature controlled system.

\subsubsection{Media preparation and pre-cultures}

Media and preculture preparation followed the same protocol as growth in static HHP vessels described in section 2.7.1. The only modification was using four serum bottles each filled with $50 \mathrm{~mL}$ of sterile reduced medium for triplicate experiments with one negative control. 
1. Decrimp the sterile reduced medium filled serum bottles before placing them into the anaerobic chamber.

2. Place the pre-cultures and sterile reduced medium in the anaerobic chamber.

\subsubsection{Powering the PUSH vessels inside the anaerobic chamber}

To maintain pre-heated temperatures during inoculation, each vessel heating system must be powered inside the anaerobic chamber. The power source here is a 12-volt rechargeable leadacid battery connected to a $1000 \mathrm{~W}$ DC to AC power converter with four AC power outlets.

1. Place the he fully charged 12 -volt battery and DC to AC power converter into the anaerobic chamber before inoculation.

2. Place heat-resistant gloves and the pre-heated PUSH vessels and heating systems into the anaerobic chamber.

3. Plug in the heating systems to the DC to AC power converter to continuously heat the vessels throughout the inoculation process (Figs. 7 and 9A).

\subsubsection{Inoculation and transfer in the anaerobic chamber}

Prior to starting the inoculation, all of the vessels, mobile heating systems, heat-resistant gloves, sterile reduced medium, and pre-cultures were transferred to the anaerobic chamber.

1. Inoculate 3 sets of 4 serum bottles each with one of the three pre-cultures to the desired cell density. Depending on growth rate and measurement technique either a $1 \%$ (v/v) inoculum volume or a target cell density of $\sim 10^{6}$ cells $/ \mathrm{ml}$ is ideal.

2. Shake each serum bottle to evenly distribute the cells.

3. Unscrew the cap on the PEEK reservoir side while maintaining sterility.

4. Remove the butyl rubber stoppers from the serum bottles and pour the medium $(\sim 50 \mathrm{~mL})$ into each of the PUSH vessels (Figs 9A\&B).

5. Screw the cap on the PEEK reservoir side to close the vessel and close the respective valve.

6. After inoculating and transferring medium into the PUSH vessels, unplug their heating systems and remove them from the anaerobic chamber.
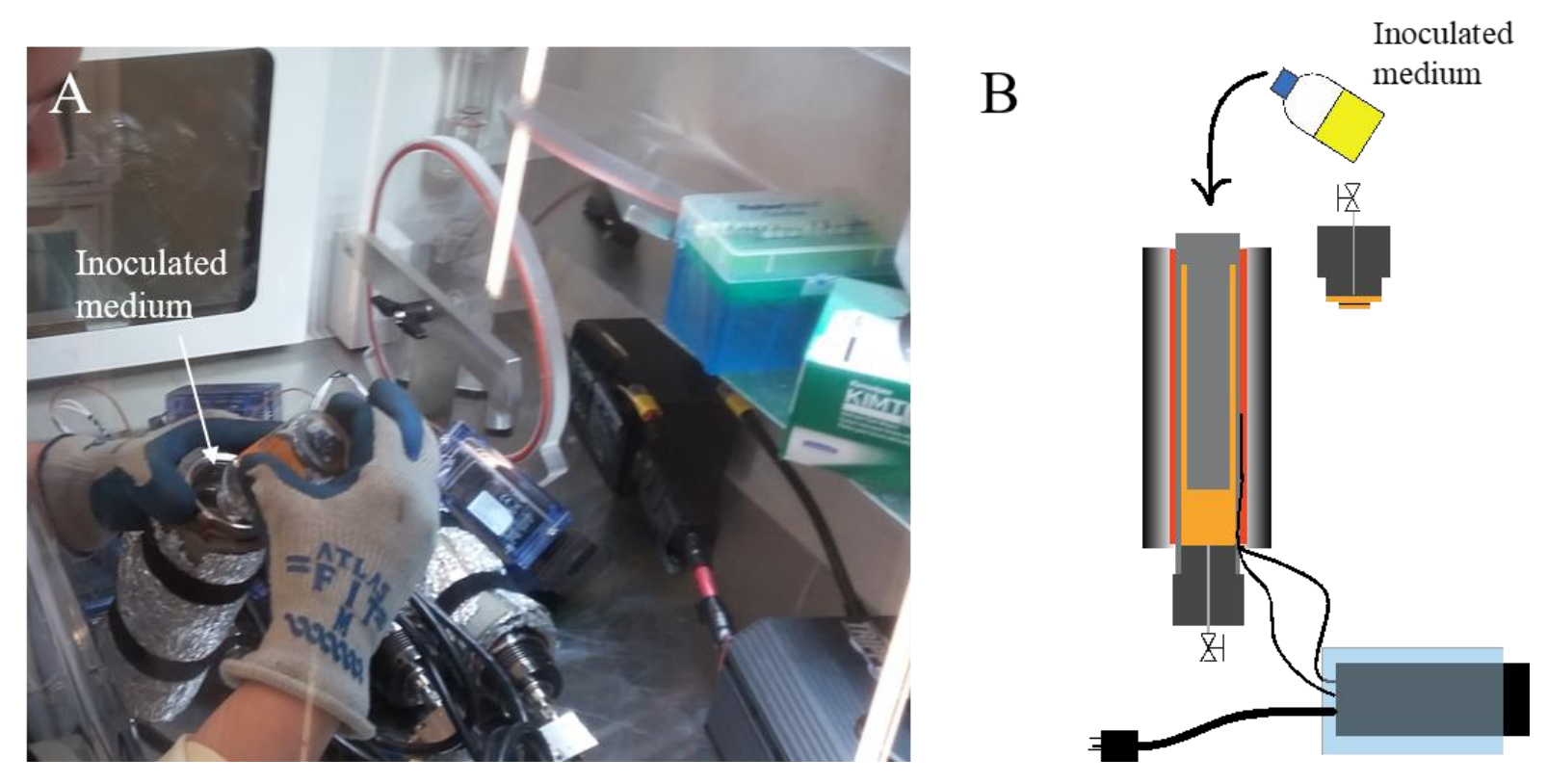
Figure 9: Picture of inoculated medium poured into a heated PUSH vessel in the anaerobic chamber (A). Three vessels were filled with inoculated medium and one vessel was filled with the sterile medium as a triplicate experiments and a negative control (A). (B) A schematic is shown on the right to reference (A).

\subsubsection{Sample pressurization}

All four vessels secured into a designated holder (Fig. 10) on the main installation frame. There, each vessel was pressurized using a HHP pump (TOP Industrie ${ }^{\odot}$ ) connected to a flexible high-pressure line made from $\mathrm{HiP}^{\odot}$ parts (Fig. 10).

1. Fill the HHP pump reservoir (reservoir size, $50 \mathrm{~mL}$ ) with water by closing the pressure valve and opening the reservoir valve. Rotate the HHP pump counterclockwise using the handles until the pump is completely filled.

2. Connect one end of high-pressure line to the HHP pump and the other end to the valve connected to the piston side on the PUSH vessel (Fig. 10).

3. Open both the pressure valve and valve to the piston on the PUSH vessel. Check to make sure the valve to the PEEK reservoir side is closed.

4. Pressurize the PUSH vessel to the target growth pressure.

5. Close the valve to the piston.

6. Repeat this procedure for all four vessels.

7. Check pressure stability regularly within the first 2-3 hours after inoculation. This is done by connecting the HHP pump to a vessel with all valves closed. Pressurize the HHP pump to the vessel pressure and only open the valve to the piston side. The pressure is stable when the pressure on the HHP pump gauge does not move when the valve to the piston side is opened.

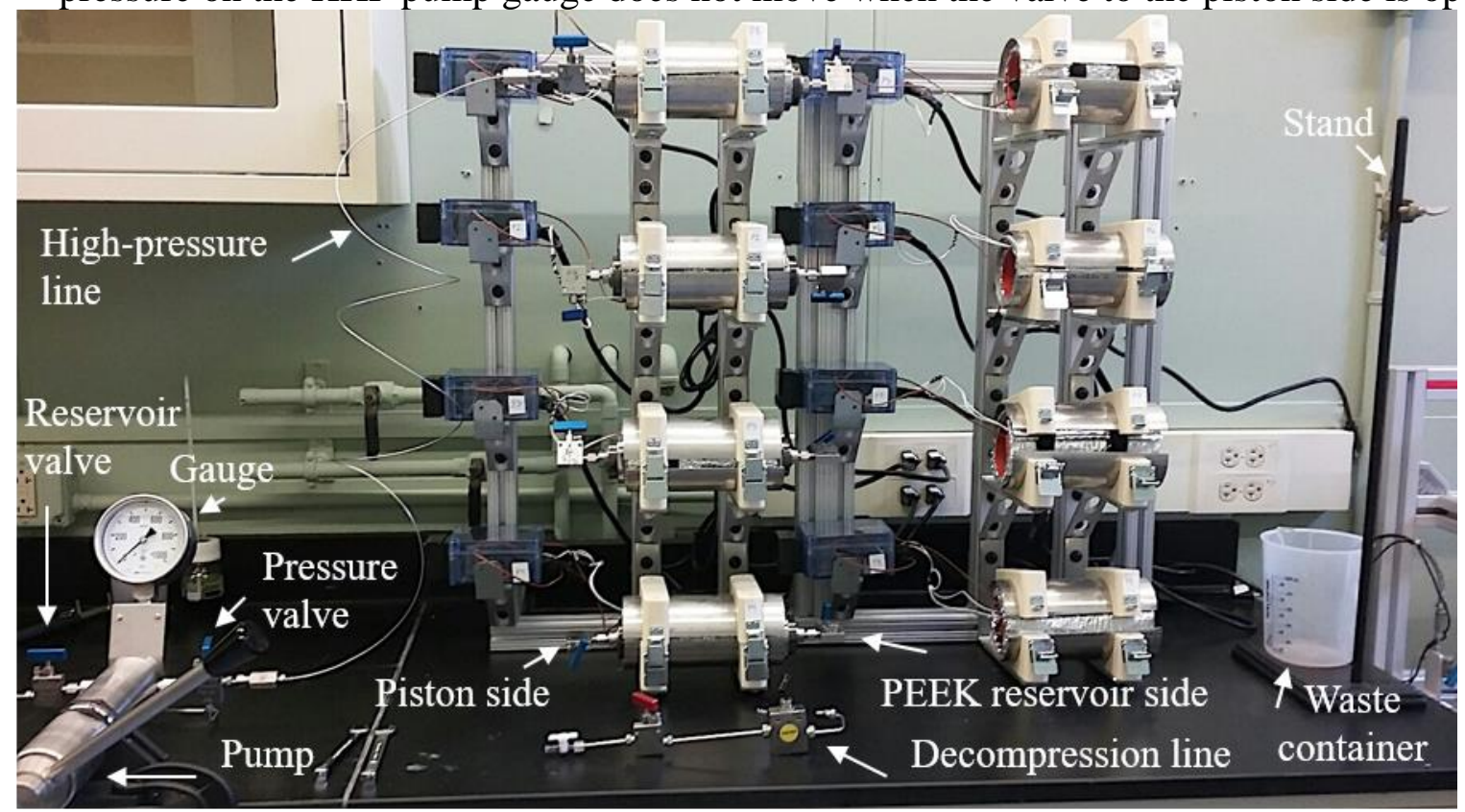

Figure 10: Picture of four heated and insulated PUSH vessels in the installation system secured in place with the manual screw pump (bottom left), high-pressure line, decompression line (bottom center), decompression line stand (right), and waste container (bottom right).

\subsubsection{Subsampling without decompressing the whole culture}

Subsampling from the PUSH vessels is done using a decompression line similar to techniques described by Foustoukos and Pérez-Rodríguez (2015). Use of the decompression line 
minimizes shear stress caused by a pressure differential during subsampling and allows for a more controlled decompression rate of the subsamples. The decompression line has two valves. The first valve is used to help pressurize the decompression line without substantial pressure loss of the vessel. The second valve is a micrometering valve used for a slow and controlled decompression rate. Cells that are initially pushed through the decompression line when the first valve is opened are discarded since they experienced extreme shear stress from rapid decompression. Additionally, the decompression line must be cleaned with ethanol and ultrapure water (18.2 $\mathrm{M} \Omega$ ) before subsampling and after sampling each vessel. Cells that come in contact with ethanol and ultrapure water $(18.2 \mathrm{M} \Omega)$ are also discarded. In total, the first 3-4 $\mathrm{mL}$ of sample is discarded each time a subsample is taken. This volume needs to be considered when planning the subsampling interval. Additionally, because the vessel installation frame stacks the vessels vertically (Fig. 6 and 10), a stand and clamp holder is used to hold and stabilize the decompression line in place while subsampling from each vessel.

1. Clean the decompression line with ethanol and sterile ultrapure water (18.2 M $\Omega$ ) water before subsampling.

2. After cleaning, check that both valves on the decompression line are closed before starting the subsampling process.

3. Connect the decompression line to the valve to the PEEK reservoir side (Fig. 11).

4. Secure the decompression line in place with a stand and clamp holder (Fig. 11).

5. Connect the high-pressure line to the valve on the piston side on the PUSH vessel (Fig. 11).

6. Fill the HHP pump reservoir with water (step 1 section 3.6.5) and then close the pump reservoir valve. Open the pressure valve and pressurize the HHP line to the pressure of the vessel.

7. Once the target pressure is reached on the HHP line, open the valve on the piston side of the PUSH vessel. Note that the pressure should not change, but should be monitored during this process.

8. Connect a sterile syringe to a luer-lok connector at the end of the decompression line (Fig. 11).

9. Next, open the first valve on the decompression line and adjust the pressure as necessary using the HHP pump. The pressure loss should be less than 5-10\%. (Fig. 11).

10. Slowly open the micrometering valve on the PEEK reservoir side and adjust the pressure if needed.

Discard the first 3-4 $\mathrm{mL}$ of fluid into a waste container.

11. Take a $0.5 \mathrm{~mL}$ aliquot of $A$. fulgidus culture, fix the cells with $2.5 \%$ gludaraldehyde, and store for analysis.

12. Once the subsample has been taken, close the valve to the PEEK reservoir opening side and disconnected the decompression line.

13. Check the pressure gauge on the HHP pump to make sure the vessel is holding the target pressure. Adjust the pressure if needed then close the valve on the piston side of the PUSH vessel.

14. Check to make sure both valves on the PUSH vessel are closed then slowly open the pump reservoir valve to release the pressure on the HHP pump.

15. Disconnect the high-pressure line from the PUSH vessel and prepare to subsample from the next vessel. 


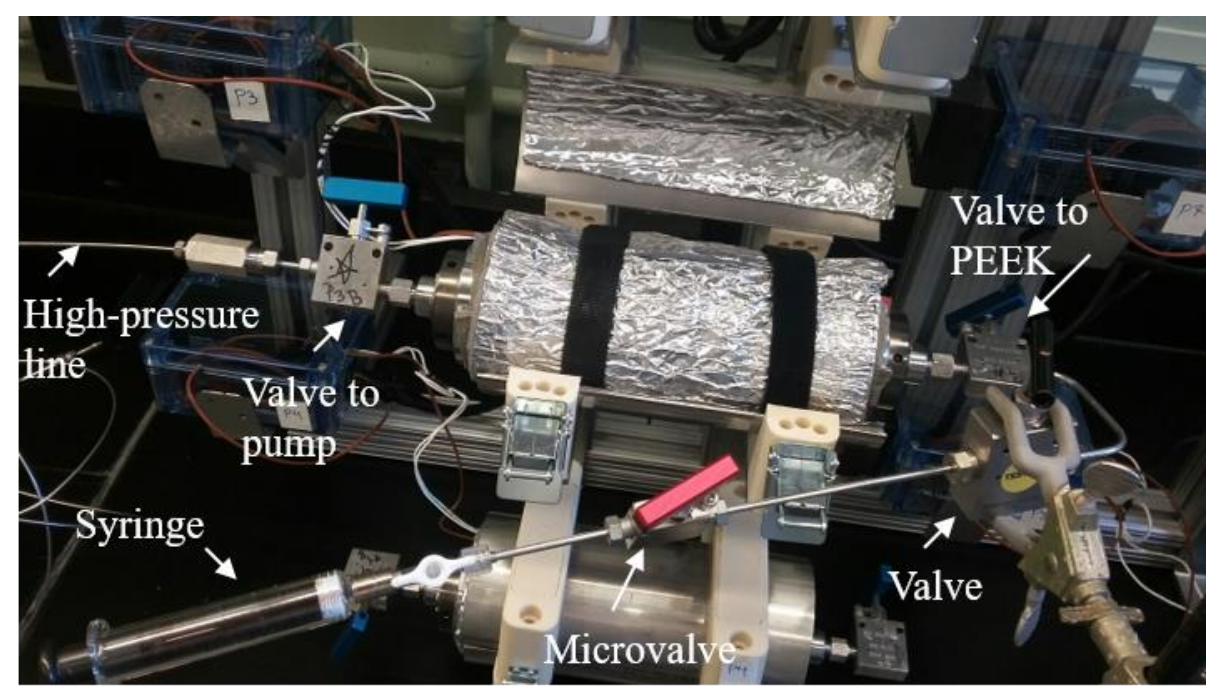

Figure 11: Picture of a heated and insulated PUSH vessel connected to a manual screw pump (left) and the decompression line (right) with a sampling syringe.

\subsection{HHP PUSH-to-PUSH transfer without decompression}

Investigating HHP microbial diversity and physiology will inevitably require transferring samples or enrichment cultures while maintaining HHP conditions. One example of such an experiment would be strain isolation from an enrichment culture, and another might be exploring the adaptation of a strain to alternate growth (e.g. pressure) conditions. Below we describe the protocol to transfer fluid from one PUSH vessel to another PUSH vessel while maintaining HHP. Choice of strain, media type, and target pressure are all customizable. Follow the protocols above to prepare and grow the culture to be transferred as well as the sterile medium in the target PUSH vessel. Modifications are described below.

1. During preparation of the PUSH vessels insert a magnetic stir bar inside each PEEK reservoir before sterilization to be used for mixing after inoculation.

2. Cut a $\sim 5$-inch long 1/8" ID high-pressure tube and fit with valve connections on both ends, including a micrometering valve on the outlet to the PUSH that will serve as the inoculum source.

3. Calibrate the fluid volume transferred in this connector (here $\sim 0.6 \mathrm{~mL}$ of fluid transferred per HHP screw pump rotation).

4. Sterilize the connector prior to installation between the two PUSH vessels and fill with sterile growth medium prior to connecting to the vessels.

5. Install the connector so that the reservoir side of the source vessel points toward the reservoir side of the target vessel (Fig. 12).

6. Connect a second HHP screw pump and gauge on the piston side of the target PUSH vessel.

7. The first step is to pressurize the fluid in the connector tube with the pump connected to the source PUSH vessel. First open the valve on the piston-side of the source PUSH vessel. While turning the pump slowly open the valve between the source PUSH vessel and the connector until the target pressure is reached.

8. Prior to inoculation pressurize the sterile growth medium in the target PUSH vessel using the second pump on the piston-side of that vessel. 
9. To transfer cells from the source PUSH to the target PUSH open the remaining valve on the connector. Turn the HHP screw pump on the source PUSH while also decreasing the pressure with the HHP screw pump on the target PUSH to slowly transfer fluid. Quantify the fluid transferred by monitoring the turns on the screw pump.

10. Following transfer, close both vessels were closed and detach from their respective HHP screw pumps.

11. Place the target PUSH on a stir plate to mix the distribute the inoculum.

12. Return the target PUSH to the frame and return to incubation temperature. Sample as needed according to the protocol above.

A.

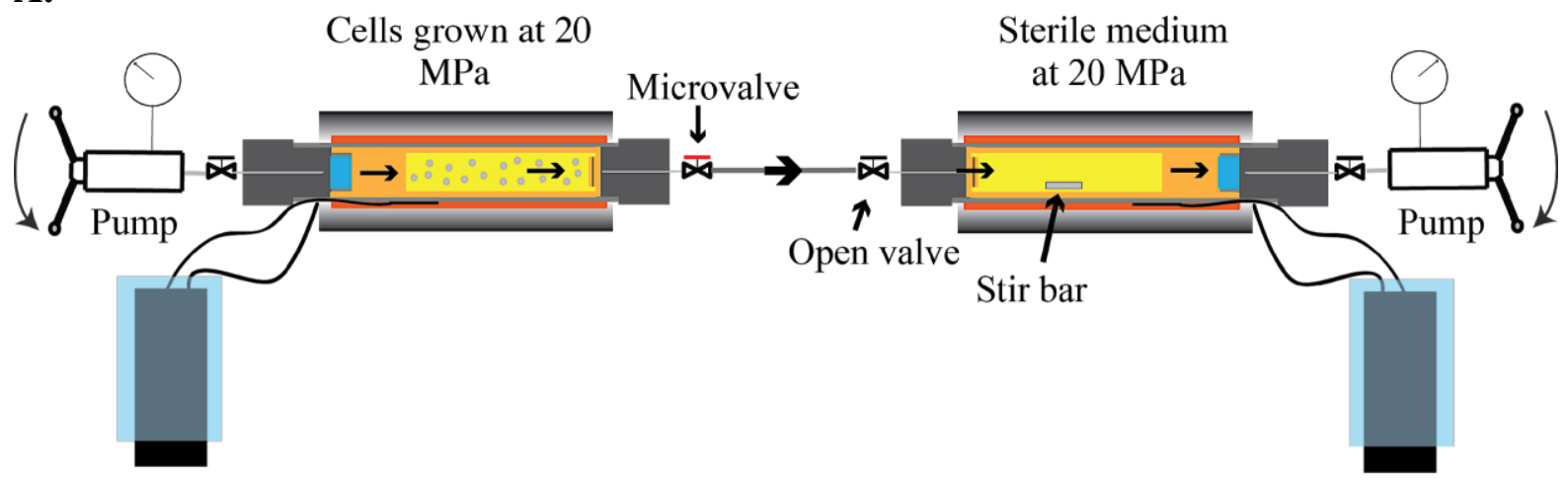

TC

B.

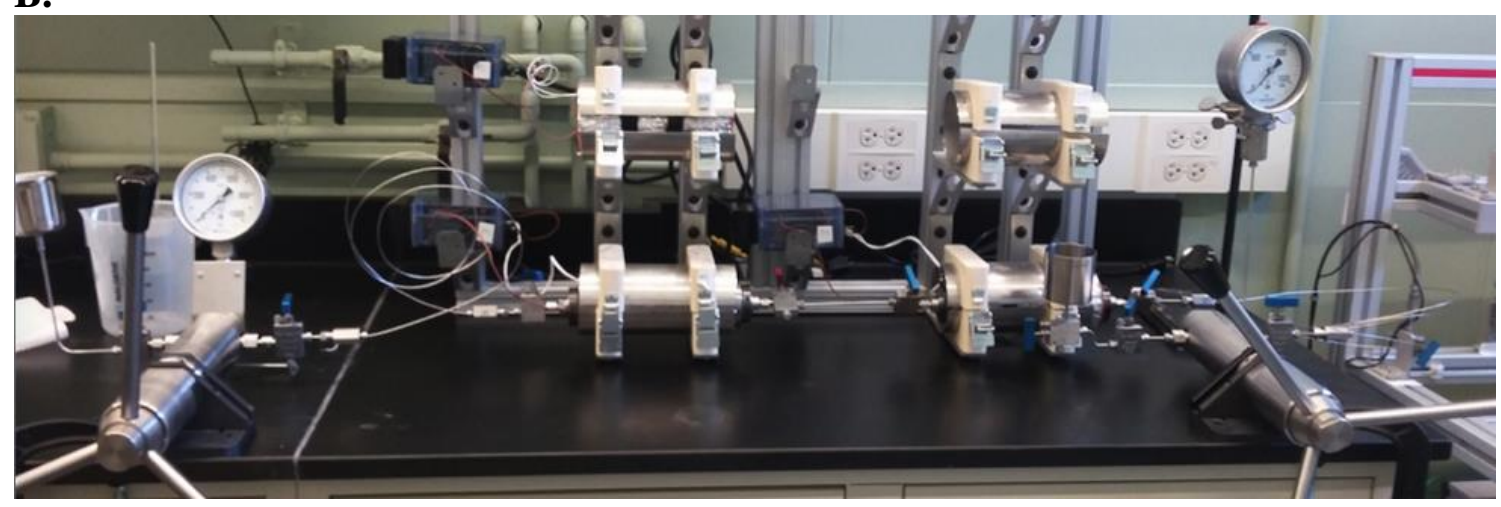

C.

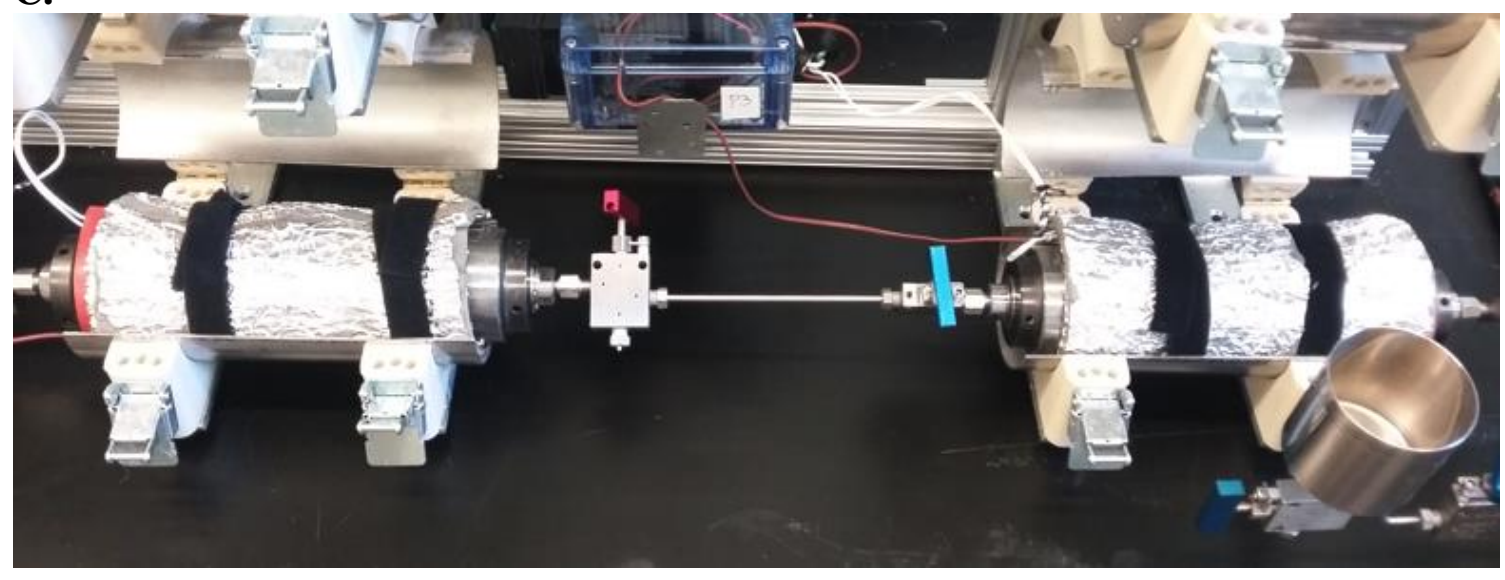


Figure 12: Schematic of the PUSH-to-PUSH transfer assembly. (A). Picture of the PUSH-to-PUSH transfer set up (B) with a zoom in on the transfer line (C).

\section{High-pressure whole-cell filtration without decompression}

The growth, survival and structural integrity of pressure-adapted microbes can be negatively impacted by decompression. The most deleterious effect is rupture of the cell envelope caused by shear stress during rapid decompression (Taylor \& Jannasch, 1976, Hemmingsen \& Hemmingsen, 1980, Park \& Clark, 2002). The extent of cell lysis is a function of the decompression rate (Taylor \& Jannasch, 1976, Park \& Clark, 2002), the presence of intracellular vacuoles (Hemmingsen \& Hemmingsen, 1980), and the style of pressurization (hydrostatic vs. hyperbaric (Park \& Clark, 2002)). For phylogenetic analyses that rely on genomic DNA extracted from whole cells, sample decompression prior to whole cell filtration and nucleic acid extraction would result in loss of nucleic acids from ruptured cells and analyses would be biased against decompression-intolerant strains. Here we present a new high-pressure filtration system developed during an oceanographic research expedition in January 2020 (Mid-Cayman Rise) to filter genomic DNA under in situ pressure conditions. The protocol outlined below assumes the fluid to be filtered is in PUSH vessel under in situ HHP. The PUSH containing the fluid to be filtered is connected to a high-pressure filter holder, followed by a back-pressure valve (BPV) (Fig. 13). For RNA-seq experiments, an alternative procedure for fixation under pressure is proposed, in order to keep the transcripts under in situ pressure conditions.

1. Using a HHP screw pump connected to the inlet of the BPV set the pressure on the BPV to just slightly above the pressure of the sample to be filtered.

2. Sterilize both sides of the filter holder with the valves attached and assemble the filter holder with a $0.2 \mu \mathrm{m}$ filter.

3. Connect the outlet of the filter holder to the BPV and the inlet to a PUSH containing sterile ultrapure (18.2 M $\Omega)$ water.

4. With a HHP screw pump attached to the piston-side of the sterile-water PUSH, fill the filter holder with sterile water and pressurize to the sample pressure. Close the valves on the filter holder and remove the PUSH containing sterile ultrapure (18.2 M $\Omega$ ) water from the assembly.

5. Connect the PUSH containing the sample to be filtered between the HHP pump and the inlet of the filter housing. Pressurize the line between the pump and the vessel containing the HHP sample.

6. While maintaining pressure in the line with the screw pump, open the valves starting at the piston-side of the PUSH and moving forward through the assembly. Be sure to maintain pressure in the line during this process (Fig. 14).

7. Once all valves are open continue turning the HHP pump to transfer the sample fluid through the line. To monitor the volume filtered collect the filtered fluid on the outlet of the BPV.

8. Once all fluid is transferred, close the valves to the filter holder and remove from the assembly.* Decompress and collect the filter in a sterile falcon tube and freeze in desired fixative until analysis.

The alternative protocol below can be used to fix the filter while still under pressure.

A3. Dedicate a specific PUSH vessel to the fixative only.

A4. Once the valves of the filter holder are closed, connect a pressure vessel (previously filled with a fixative, which volume corresponds to half of the filtrated volume) between the HHP pump and the inlet of the filter housing. 
A5. Pressurize the fixative vessel at the corresponding pressure. Then follow the procedure starting at step 6 .

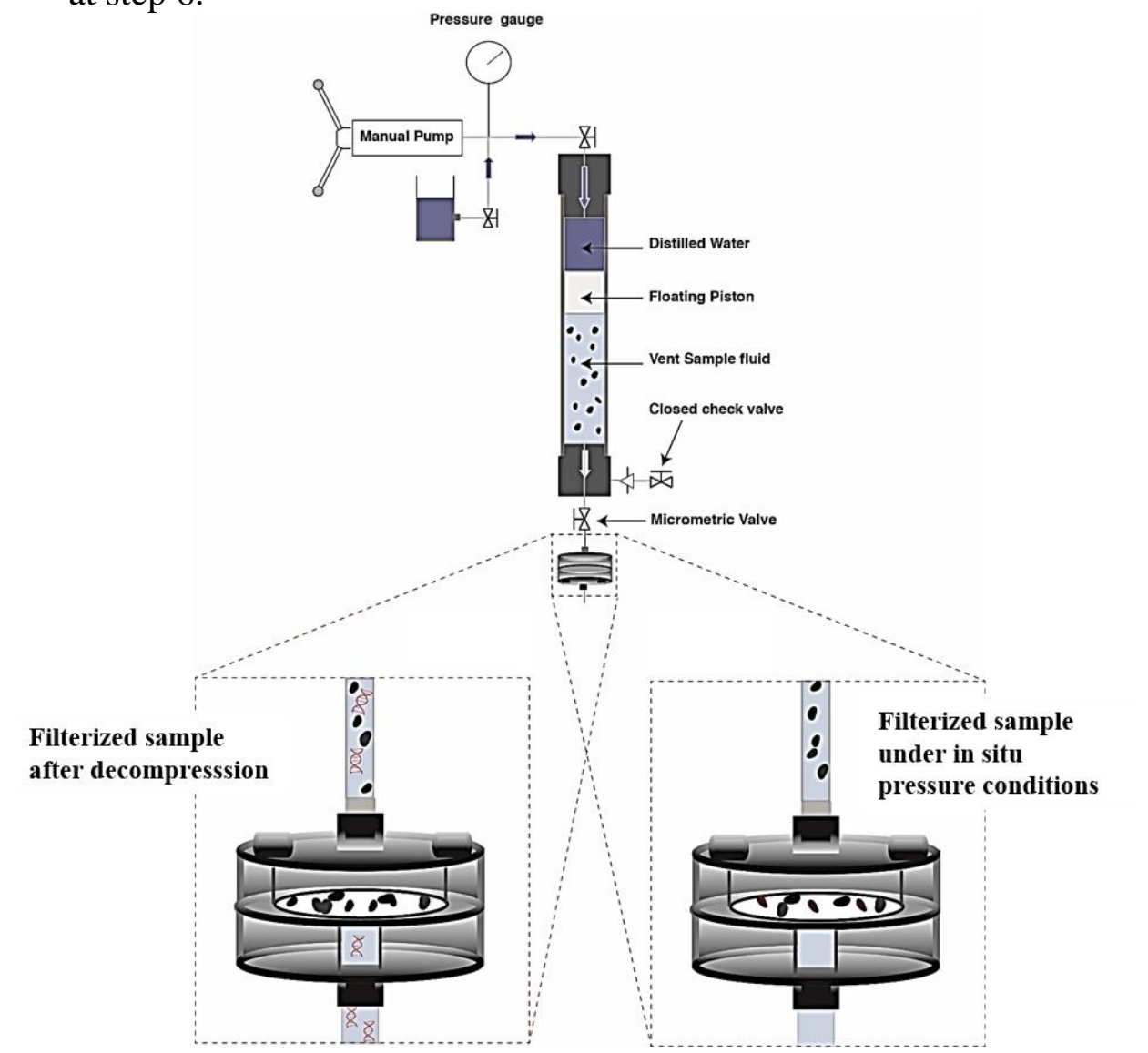

Figure 13: Schematic of the high-pressure filtration assembly connected to a floating-variable piston (e.g. PUSH for whole-cell filtration without decompression.

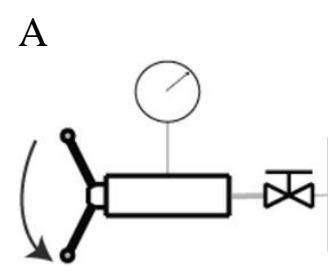

Manual Pump

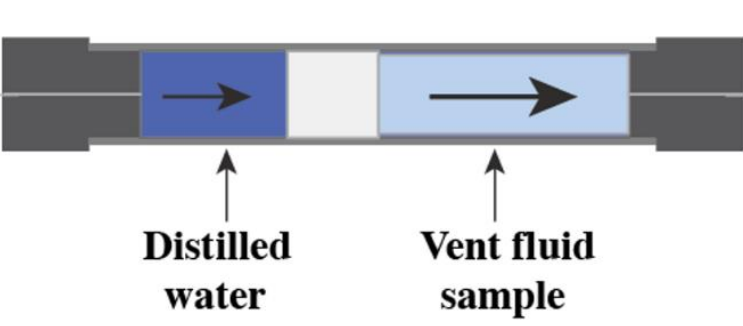

water

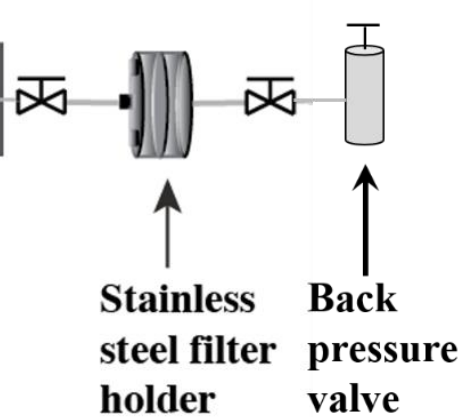

A.

B. 


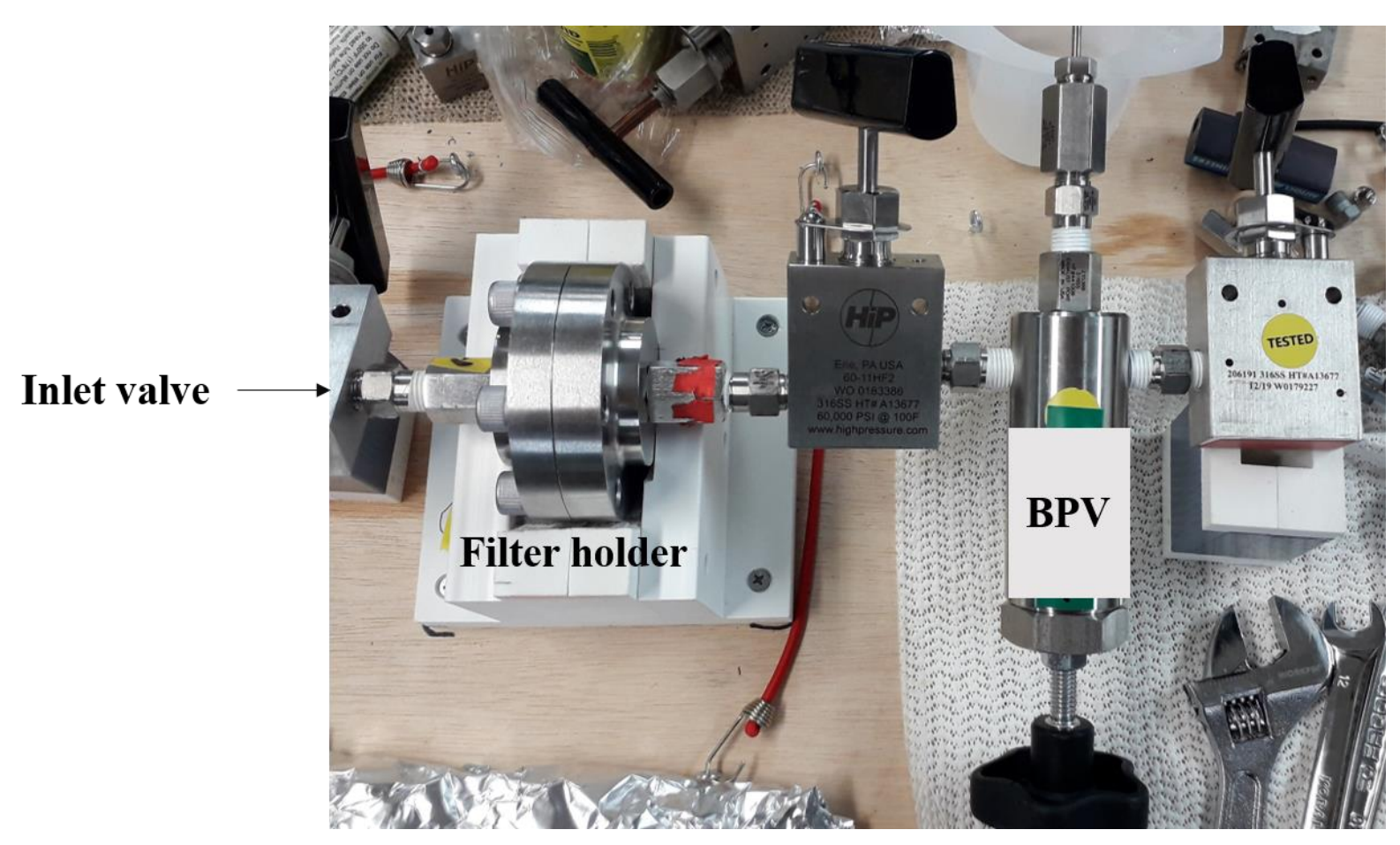

Figure 14: Schematic of the PUSH connected to the pressure holder and the backpressure valve (A). Picture of the pressure holder and the backpressure valve (BPV) (B).

\section{Concluding remarks}

Here, we present two high temperature (HT) and high hydrostatic pressure (HHP) batch cultivation systems and example protocols for growing anaerobic thermophiles under batch conditions. This was done to ease the adoption of HT-HHP techniques to more laboratories and expand our understanding of extremophiles from deep-sea vents and hot subseafloor environments under natural conditions. Previous HT-HHP microbial cultivation has highlighted the fact that relatively little is known about the physiology and metabolism of deep-sea and subseafloor microorganisms. Fluctuating temperature, pressure, and geochemical conditions of growth all have an impact on microbial functionality. To better understand the range of growth and adaptive strategies deep-sea and subseafloor microorganisms have under in situ conditions, a large effort is needed to continue to grow these extremophiles under various HT-HHP and geochemical conditions.

\section{Conflict of interest}

The authors declare that the research was conducted in the absence of any commercial or financial relationships that could be construed as a potential conflict of interest.

\section{Author Contribution}

GCO, AC, and KLR designed this research project. GCO wrote the original draft; All authors contributed to revisions of the manuscript, figures and approved the submitted version.

\section{Funding}


Funding for this work was provided by the NASA (Exobiology Program: NNX13AP2G9 and PSTAR: 80NSSC17K0252 to KLR), the Deep Carbon Observatory (Subawards: 10371-07, 10561-01, and 10311-11 to KLR), an NSF Graduate Fellowship (FAIN 1247271 and 1744655 to GCO) and a GSA Research Grant to GCO. Additional support was provided by startup funds from Rensselaer Polytechnic Institute to KLR.

\section{Acknowledgements}

We would like to give our many thanks Dr. E. Bruce Watson for gifting the Habitability and Extreme Life Laboratory (HELL) four high-pressure vessels that were used for HT-HHP cultivation with sample decompression. We would like to thank Christopher Hoff for his help with the thermal control system.

\section{References}

Baross, J. a, \& Deming, J. W. (1983). Growth of 'black smoker' bacteria at temperatures of at least $250{ }^{\circ} \mathrm{C}$. Nature, 303(5916), 423-426.

Bowles, M. W., Samarkin, V. a., \& Joye, S. B. (2011). Improved measurement of microbial activity in deep-sea sediments at in situ pressure and methane concentration. Limnology and Oceanography: Methods, 9, 499-506.

Canganella, F., Jones, W. J., Gambacorta, A., \& Antranikian, G. (1997). Biochemical and phylogenetic characterization of two novel deep-sea Thermococcus isolates with potentially biotechnological applications. Archives of Microbiology, 167(4), 233-238.

Cario, A., Oliver, G. C., \& Rogers, K. L. (2019). Exploring the deep marine biosphere: challenges, innovations, and opportunities. Frontiers in Earth Science, 7, 225.

Deming, J. W., \& Colwell, R. R. (1981). Barophilic bacteria associated with deep-sea animals. Bioscience, 31(7), 507-511.

D'Hondt, S. (2004). Distributions of Microbial Activities in Deep Subseafloor Sediments. Science, 306(5705), 2216-2221.

Foustoukos, Dionysis I., and Ileana Pérez-Rodríguez (2015). A Continuous Culture System for Assessing Microbial Activities in the Piezosphere. Applied and Environmental Microbiology 81, no. 19: 685056.

Garel, M., Bonin, P., Martini, S., Guasco, S., Roumagnac, M., Bhairy, N., \& Tamburini, C. (2019). Pressure-retaining sampler and high-pressure systems to study deep-sea microbes under in situ conditions. Frontiers in microbiology, 10, 453.

Hemmingsen, B. B., \& Hemmingsen, E. A. (1980). Rupture of the cell envelope by induced intracellular gas phase expansion in gas vacuolate bacteria. Journal of bacteriology, 143(2), 841-846.

Jannasch, H. W., Cuhel, R. L., Wirsen, C. O., \& Taylor, C. D. (1980). An approach for in situ studies of deep-sea amphipods and their microbial gut flora. Deep Sea Research Part A. Oceanographic Research Papers, 27(10), 867-872.

Jannasch, H. W., Wirsen, C. O., \& Doherty, K. W. (1996). A pressurized chemostat for the study of marine barophilic and oligotrophic bacteria. Applied and environmental microbiology, 62(5), 1593-1596.

Kato, C. (2006). Handling of Piezophilic Microorganisms, 35(5).

Maier, R. M. (2009). Bacterial Growth. Environmental Microbiology, 37-54.

Marietou, A., Nguyen, A. T. T., Allen, E. E., \& Bartlett, D. H. (2014). Adaptive laboratory evolution of Escherichia coli K-12 MG1655 for growth at high hydrostatic pressure. Frontiers in Microbiology, 5(DEC), 1-8.

Marteinsson, V. T., Birrien, J. L., Raguenes, G., da Costa, M. S., \& Prieur, D. (1999). Isolation and characterization of Thermus thermophilus Gy1211 from a deep-sea hydrothermal vent. Extremophiles, 3(4), 247-251.

McNichol, J., Sylva, S. P., Thomas, F., Taylor, C. D., Sievert, S. M., \& Seewald, J. S. (2016). Assessing 
microbial processes in deep-sea hydrothermal systems by incubation at in situ temperature and pressure. Deep Sea Research Part I: Oceanographic Research Papers, 115, 221-232.

McNichol, J., Stryhanyuk, H., Sylva, S. P., Thomas, F., Musat, N., Seewald, J. S., \& Sievert, S. M. (2018). Primary productivity below the seafloor at deep-sea hot springs. Proceedings of the National Academy of Sciences, 115(26), 6756-6761

Oliver, G. C., Cario, A., \& Rogers, K. L. (2020). Rate and Extent of Growth of a Model Extremophile, Archaeoglobus fulgidus, Under High Hydrostatic Pressures. Frontiers in microbiology, 11, 1023.

Park, C. B., \& Clark, D. S. (2002). Rupture of the cell envelope by decompression of the deep-sea methanogen Methanococcus jannaschii. Applied and environmental microbiology, 68(3), 1458-1463.

Parkes, R. J., Sellek, G., Webster, G., Martin, D., Anders, E., Weightman, A. J., \& Sass. (2009). Culturable prokaryotic diversity of deep, gas hydrate sediments: first use of a continuous high-pressure, anaerobic, enrichment and isolation system for subseafloor sediments (DeepIsoBUG). Environmental Microbiology, 11(12), 3140-3153.

Parkes, R. J., Cragg, B., Roussel, E., Webster, G., Weightman, A., \& Sass, H. (2014). A review of prokaryotic populations and processes in sub-seafloor sediments, including biosphere: Geosphere interactions. Marine Geology, 352, 409-425.

Schwarz, J. R., Yayanos, A. A., \& Colwell, R. R. (1976). Metabolic activities of the intestinal microflora of a deep-sea invertebrate. Applied and Environmental Microbiology, 31(1), 46-48.

Takai, K., Nakamura, K., Toki, T., Tsunogai, U., Miyazaki, M., Miyazaki, J., et al. (2008). Cell proliferation at $122 \mathrm{C}$ and isotopically heavy $\mathrm{CH} 4$ production by a hyperthermophilic methanogen under highpressure cultivation. Proceedings of the National Academy of Sciences, 105(31), 10949-10954.

Tamburini, C., Garcin, J., and Bianchi, A. (2003). Role of deep-sea bacteria in organic matter mineralization and adaptation to hydrostatic pressure conditions in the NW Mediterranean Sea, 32, 209-218.

Tamburini, C. (2006). Life under pressure. Deep-sea microbial ecology. Life as we know it. Series: Cellular Origin and Life in Extreme Habitats and Astrobiology. Springer, Dordrecht, The Netherlands pp. 125-143

Tasumi, E., Yanagawa, K., Miyazaki, J., \& Takai, K. (2015). In Vitro High-Pressure Incubation and Activity Measurement of Deep-Sea Methanogenic Archaea, (June), 51-64.

Taylor, C. D., \& Jannasch, H. W. (1976). Subsampling technique for measuring growth of bacterial cultures under high hydrostatic pressure. Applied and environmental microbiology, 32(3), 355-359.

Yayanos, A. A. (1995). Microbiology to 10,500 meters in the deep sea. Annual review of microbiology, 49, $777-805$.

Yayanos, A. A. (1986). Evolutional and ecological implications of the properties of deep-sea barophilic bacteria. Proceedings of the National Academy of Sciences, 83(24), 9542-9546.

Yayanos, A. A. (2001). 30 Deep-sea Piezophilic Bacteria. Methods in Microbiology, 30, 615-637.

Yayanos, A. A., Dietz, A. S., \& Boxtel, R. V. A. N. (1982). of Deep-Sea Bacteria, 44(6), 1356-1361.

Zobell, C. E., \& Oppenheimer, C. H. (1950). Some effects of hydrostatic pressure on the multiplication and morphology of marine bacteria. Journal of Bacteriology, 60(0), 771-781. 